\title{
Inhibitory Effects of Endogenous Linoleic Acid and Glutaric Acid on the Renal Glucuronidation of Berberrubine in Mice and on Recombinant Human UGT1A7, 1A8, and 1A9s
}

\author{
Na Yang, Sijia Li, Caixia Yan, Runbin Sun, Jun He, Yuan Xie, Ying Peng, Guangji Wang, \\ and Jiye Aa
}

\begin{abstract}
Jiangsu Province Key Laboratory of Drug Metabolism and Pharmacokinetics, State Key Laboratory of Natural Medicines, Jiangsu Province Key Laboratory of Drug Design and Optimization, China Pharmaceutical University, Nanjing, People's Republic of China
\end{abstract}

Received September 18, 2017; accepted January 8, 2018

\begin{abstract}
Berberrubine (BRB) has a strong lipid-lowering effect and can be extensively metabolized into berberrubine-9-O- $\beta$-D-glucuronide (BRBG) in vivo. Recently, pharmacokinetics studies showed that the production of BRBG was significantly decreased in the urine of mice fed with a high-fat diet (HFD), indicating a decreased glucuronidation capacity. Based on the UDPglucuronosyltransferase (UGT) isoform identification, hepatic and renal microsomal incubation, glucuronidation was examined to suggest the metabolism of BRB in liver and kidneys. The results showed that the renal UGT activity for metabolizing BRB markedly decreased, which may be highly related to the decreased expression and activity of renal Ugt1a7c. Surprisingly, in vitro studies revealed neither BRB nor BRBG inhibited the renal UGT activity. By employing an integrated strategy of
\end{abstract}

metabolomics and pharmacokinetics, we identified and confirmed for the first time the inhibitory effect of some potential endogenous molecules on the renal glucuronidation of C57BL/6J mice, such as glutaric acid (GA) and linoleic acid (LA). By employing recombinant human UGTs, we found that GA and LA efficiently affect the activity of recombinant human UGT1A7, 1A9, and $1 A 8$ at their normal or abnormal physiologic levels in vivo. GA (2 mM) markedly inhibited the activity of UGT1A7 by $89.4 \%$ and UGT1A9 by $32.8 \%$. The inhibition rates reached $99.3 \%$ for UGT1A9, $48.3 \%$ for UGT1A7, and $46.8 \%$ for UGT1A8 with LA at $200 \mu \mathrm{M}$. It has been suggested that the endogenous molecules have the potential to affect the efficiency of glucuronidation, which might be a key factor contributing to individual differences in drug metabolism.

\section{Introduction}

Drugs or other xenobiotics usually undergo two phases of biotransformation, which determine their pharmacokinetic profiles, therapeutic effects, and toxic side effects (Guengerich, 2006; Oda et al., 2015). As one of the most important phase II reactions, glucuronidation is responsible for $\sim 35 \%$ of all drug metabolism by phase II enzymes (Kiang et al., 2005). The toxicity of many toxic xenobiotics could be attenuated during

This study was financially supported by the National Natural Science Foundation of the People's Republic of China [81573495, 81530098 and 81673679], the National Key Special Project of Science and Technology for Innovation Drugs of China [2015ZX09501001], the Natural Science Foundation of Jiangsu Province [BL2014070], the Project for Jiangsu Province Key Laboratory of Drug Metabolism and Pharmacokinetics [BM2012012], the project of university collaborative innovation center of Jiangsu province (Modern Chinese medicine center and biological medicine center).

https://doi.org/10.1124/mol.117.110668.

S This article has supplemental material available at molpharm. aspetjournals.org. the metabolic process in vivo, and many of them are dependent on the glucuronidation process for detoxification (Tephly and Burchell, 1990). For instance, 7-ethyl-10-hydroxycamptothecin (SN-38), a metabolite of irinotecan, is correlated to severe toxicities, including diarrhea and leucopenia, and it could be detoxified after conjugation with glucuronic acid (van der Bol et al., 2011). Benzo[a]pyrene, a dangerous inducer related to lung cancer, could also be detoxified through glucuronidation (Kua et al., 2012). Hence, the disturbance of the glucuronidation process is closely related with drug-induced toxicity or the risk of some diseases.

In most cases, the capacity of drug glucuronidation is dependent on the activity of UDP-glucuronosyltransferases (UGTs). The mammalian UGT superfamily can be divided into UGT1 (1A), and UGT2 (2A, 2B) families. Previous studies have reported that the expression or activity of UGTs can be affected by many factors involving nuclear receptors, diverse diseases, inflammation, oxidative stress, and other pathologic or abnormal physiologic effects (Gradinaru et al., 2012; Xu

ABBREVIATIONS: 4-MU, 4-methylumbelliferone; $\mathrm{AUC}_{0-\mathrm{t}}$, area under the plasma concentration-time curve calculated to the last measured concentration; BRB, berberrubine; BRBG, berberrubine-9-O- $\beta$-D-glucuronide; $C E$, collision energy; $C M B$, control diet with multiple doses of BRB; CMC-Na, carboxymethyl cellulose sodium salt; CSB, control diet with a single dose of BRB; DDls, drug-drug interactions; DP, declustering potential GA, glutaric acid; HFD, high-fat diet; HMB, high-fat diet with multiple dose of BRB; HMDB, Human Metabolome Database; IS, internal standard; LA, linoleic acid; LC-MS/MS, liquid chromatography with tandem mass spectrometry; MPA, mycophenolic acid; MPAG, mycophenolic acid glucuronide; $\rho N P G, \rho$-nitrophenyl glucuronide; PCR, polymerase chain reaction; PLS-DA, partial least squares discriminant analysis; PPAR $\alpha$, peroxisome proliferator-activated receptor $\alpha$; SN-38, 7-ethyl-10-hydroxycamptothecin; UDP-GLcA, uridine diphosphate glucuronic acid; UGDH, UDP-glucose 6-dehydrogenase; UGP, UDP-glucose pyrophosphorylase; UGT, UDP-glucuronosyltransferase. 
et al., 2012; Gruber et al., 2013; Xie et al., 2013). For instance, the relative mRNA levels of partial UGT isoforms were influenced by steatosis induced by obesity (Xu et al., 2012). The glucuronidation of $\beta$-estradiol and 4-methylumbelliferone in the kidneys was significantly reduced in chronic renal insufficiency rats (Yu et al., 2006).

It is well known that disease states or other abnormal physiologic states are generally accompanied with complicated changes in internal homeostasis involving endogenous molecules, enzymes, metabolic pathways, and signaling pathways. However, the direct influence of endogenous molecules with altered levels in those processes are rarely reported, and some potential endogenous molecules may be highly related with altered activity of UGT isoforms; for example, the activities of UGT1A1, 1A6, and 1A7 are altered in rats with diabetes, accompanied by disorders of the metabolism of carbohydrates, protein, and fatty acids (Xie et al., 2013). As a result of their remarkable significance in clinical drug safety, these drug-drug interactions (DDIs) have drawn an extensive attention. Many drugs have been reported to exert inhibitory or inducing effects on UGT isoforms, which can affect glucuronidation and cause DDIs. However, little is known about the effect of endogenous molecules on UGT isoforms.

In addition to being located in the liver, UGT isoforms are also distributed in the kidneys, gastrointestinal tract, brain, and some other tissues (Court et al., 2012; Rowland et al., 2013). Some previous studies have indicated that UGT isoforms are highly expressed in the kidneys, second only to their expression in the liver (Lohr et al., 1998; Kerdpin et al., 2008; Mutsaers et al., 2013). Because the kidneys possess a high blood flow ( $\sim 25 \%$ cardiac output) and participate in the formation of urine (Atherton, 2012), the glucuronidation of drugs in the kidneys is extremely important for drug metabolism and elimination. Meanwhile, the kidney is exposed to many endogenous or exogenous molecules, which may influence the activity of renal UGT isoforms.

Berberrubine (BRB), an active lipid-lowing metabolite of berberine (Li et al., 2010; Zhou et al., 2014), showed a high proportion of glucuronidation metabolism in vivo in our previous study (Yang et al., 2017a). In our present study, for the first time, based on the specific metabolic pattern of BRB, our data suggested that some important endogenous molecules are potentially effective endogenous inhibitors on mouse renal glucuronidation via the employment of an integrated strategy of metabolomics and pharmacokinetics. Considering the species differences in UGT isoforms, and to provide more evidence for clinical research, we used recombinant human UGT isoforms to evaluate these potential endogenous inhibitors.

This study provides important evidence that some important endogenous molecules could disturb the renal glucuronidation process and influence the activity of several UGT isoforms, which could be a constructive example demonstrating the interaction of endogenous molecules on drug-metabolizing enzymes.

\section{Materials and Methods}

Reagents. BRB (purity >95\%) was synthetized by Chemzam Pharmtech (Nanjing, People's Republic of China), and berberrubine9-O- $\beta$-D-glucuronide (BRBG) was prepared and identified by the Key Laboratory of Drug Metabolism and Pharmacokinetics at China Pharmaceutical University (Yang et al., 2017a). The control diet (AIN-93M) and high-fat diet (HFD, 60\% calories from fat and 1\% cholesterol) were purchased from Trophic Animal Feed High-Tech (Nantong, People's Republic of China). We purchased $\rho$-nitrophenol $(\rho \mathrm{NP}), \rho$-nitrophenyl glucuronide ( $\rho \mathrm{NPG}$ ), UDP-glucuronic acid (UDP-GLcA), alamethicin, Dsaccharic acid 1,4-lactone, 4-methylumbelliferone (4-MU), $\beta$-estradiol, naloxone, mycophenolic acid (MPA), glutaric acid (GA), linoleic acid (LA), hydroxyglutaric acid, urea, aminoisobutyric acid, alanine, 3-hydroxybutyric acid, palmitic acid, stearic acid, taurine, and glyceric acid from Sigma-Aldrich (St. Louis, MO). The stableisotope-labeled internal standard (IS) compound myristic acid$1,2-{ }^{13} \mathrm{C}_{2}$ (99 atom $\%{ }^{13} \mathrm{C}$ ), methoxyamine hydrochloride (purity $98 \%$ ), pyridine ( $\geq 99.8 \%$ gas chromatographic), $N$-methyl- $N$-trimethylsilyltrifluoroacetamide, and $1 \%$ trimethylchlorosilane were also provided by Sigma-Aldrich. Recombinant human UGT isoforms were purchased from BD Biosciences (San Jose, CA).

Pharmacokinetic Studies. We purchased C57BL/6J mice (male; 6 weeks old; weighing 18-22 g) from the College of Animal Science and Technology (Yangzhou University, Yangzhou, People's Republic of China), and they were housed under a 12-hour light/dark cycle with free access to food and water (temperature, $22 \pm 3^{\circ} \mathrm{C}$; humidity, $55 \% \pm$ $5 \%$ ). All animal studies were performed with the approval of the animal ethics committee of China Pharmaceutical University. The mice were randomly divided into CSB (control diet with a single dose of BRB), CMB (control diet with multiple doses of BRB), and HMB (high-fat diet with multiple dose of BRB) groups. Over 6 consecutive weeks, the mice in the CMB and HMB groups were intragastrically administered BRB ( $50 \mathrm{mg} / \mathrm{kg}$ per day), and the mice in the CSB group were gavaged with vehicle carboxymethyl cellulose sodium salt (CMC$\mathrm{Na}, 5 \%)$ as a control. All mice were fasted overnight and were given free access to water ( 12 hours) before the experiments. Blood samples from all mice were collected into heparinized tubes at $0.083,0.25,0.5$, $1,1.5,2,4,6$, and 8 hours after the last BRB administration $(50 \mathrm{mg} / \mathrm{kg})$. Similarly, urine samples were collected in the metabolic cages for 12 hours after the last administration. All samples were prepared in the manner previously reported elsewhere (Yang et al., 2016).

Measurement of UGT Activity and UDP-GLcA Levels in the Liver and Kidneys of C57BL/6J Mice. C57BL/6J mice (male; 6 weeks old; weighing 18-22 g) were randomly divided into four groups: $\mathrm{C}$ (control diet), $\mathrm{CB}$ (control diet with $\mathrm{BRB}$ administration), $\mathrm{H}$ (high-fat diet), HB (high-fat diet with BRB administration). The mice were housed under a 12-hour light/dark cycle with free access to food and water (temperature, $22 \pm 3^{\circ} \mathrm{C}$; humidity, $55 \% \pm 5 \%$ ). All animal studies were performed with the approval of the animal ethics committee of China Pharmaceutical University. The mice in the CB and $\mathrm{HB}$ groups were intragastrically administered $50 \mathrm{mg} / \mathrm{kg}$ of BRB for 6 consecutive weeks, and the mice in the $\mathrm{C}$ and $\mathrm{H}$ groups were gavaged with vehicle CMC-Na (5\%) as controls. The body weights of the mice were recorded every week.

UGT enzyme assays were performed as per the previous method (Liu et al., 2013; Wang et al., 2015). Hepatic and renal microsomes were prepared from the $\mathrm{C} 57 \mathrm{BL} / 6 \mathrm{~J}$ mice according to the method described previously elsewhere (Feere et al., 2015). Hepatic and renal microsomes of C57BL/6J mice from the four groups were preincubated with alamethicin for 30 minutes at $4^{\circ} \mathrm{C}$. The incubation contained microsomes $(0.2 \mathrm{mg} / \mathrm{ml}$ protein), $50 \mathrm{mM}$ Tris-HCl buffer ( $\mathrm{pH} 7.4$ ), $20 \mu \mathrm{g} / \mathrm{ml}$ alamethicin, $2 \mathrm{mM}$ UDP-GLcA, $10 \mathrm{mM} \mathrm{MgCl}_{2}, 5 \mathrm{mM}$ Dsaccharic acid 1,4-lactone, and $50 \mu \mathrm{M}$ BRB at $37^{\circ} \mathrm{C}$ for 1 hour. Similarly, the UDP-GLcA levels were determined in the incubation system according to methods previously described elsewhere (Bánhegyi et al., 1996; Yamamura et al., 2000; Kang et al., 2010) based on the formation of $\rho$ NPG. All reactions were terminated by icecold acetonitrile (containing $10 \mathrm{ng} / \mathrm{ml}$ IS) at a ratio of 1:3 (v/v) and prepared for liquid chromatography with tandem mass spectrometry (LC-MS/MS) analysis.

Identification of UGT Isoenzymes. The total RNA of the liver and kidneys was isolated and reverse-transcribed into cDNA based on a method previously described by Mannhalter et al. (2000). Quantitative real-time polymerase chain reaction (PCR) was used to 
determine the relative mRNA levels of the UGT isoenzymes. Based on previous methods (Livak and Schmittgen, 2001; Margaillan et al., 2015), the quantitative PCR data for each UGT $\left(\mathrm{Ct}_{\mathrm{UGT}}\right)$ were first normalized with an internal standard $\left(\Delta \mathrm{Ct}_{\mathrm{UGT}}\right)$, and then normalized with $\Delta \mathrm{Ct}$ values $\left(\Delta \mathrm{Ct}_{\text {Low }}\right)$ of the sample expressing the lowest levels of UGT isoforms to determine $\Delta \Delta \mathrm{Ct}_{\mathrm{UGT}}$. The resulting value of $2^{-\Delta \Delta \mathrm{Ct} \text { UGT }}$ was then used to determine the relative quantification of UGT isoforms.

Competitive UGT inhibitory effects were determined in vitro to confirm the subtype of UGT isoforms in glucuronidation of BRB using methods previously described elsewhere (Liu et al., 2013; Wang et al., 2015) with a slight modification. Then, $50 \mu \mathrm{M}$ BRB and different concentrations of MPA (40, 200, or $1000 \mu \mathrm{M}, n=3)$ were simultaneously incubated in blank renal microsomes. The incubation system and processing method were the same as the UGT activity assay mentioned earlier. The amount of BRBG was measured.

The renal microsomes of the four groups $(\mathrm{C}, \mathrm{CB}, \mathrm{H}$, and $\mathrm{HB})$ were prepared and individually incubated with $1 \mathrm{mM}$ MPA for 30 minutes; the other constituents of this incubation system were the same as previously mentioned. The amount of mycophenolic acid glucuronide (MPAG) formation was quantified by LC-MS/MS. The direct inhibition effects of BRB or BRBG on MPA metabolism were also evaluated by coincubating MPA with BRB or BRBG ( $50 \mu \mathrm{M}$, respectively, $n=3)$ in blank renal microsomes.

Metabolomic Study. The urine metabolomic study was performed according to the well-developed metabolic platform based on the gas chromatography/mass spectrometry technique, as described previously elsewhere (Aa et al., 2005; Gu et al., 2015). Briefly, $30 \mu \mathrm{l}$ of urine samples were preincubated with $30 \mu \mathrm{l}$ of urease $(10 \mathrm{mg} / \mathrm{ml})$ at $37^{\circ} \mathrm{C}$ for 1 hour. The incubation was terminated with methanol (containing $5 \mu \mathrm{g} / \mathrm{ml}$ IS $\left[{ }^{13} \mathrm{C}_{2}\right]$-myristic acid) at a ratio of $1: 4(\mathrm{v} / \mathrm{v})$ and vortexed for protein precipitation. An aliquot of $100 \mu \mathrm{l}$ supernatant was dried and derivatized using the same method as was used for the plasma samples (Guo et al., 2016). The raw data were processed, and the endogenous compounds were identified based on previous methods (Aa et al., 2005).

Evaluation of Inhibitory Capability of the Typical Endogenous Molecules on UGT Isoforms. The concentrations of the compounds administered are designed and calculated according to the physiologic level reported in the Human Metabolome Database (HMDB) as follows: $\mathrm{LA}=$ low dose, $2 \mu \mathrm{M}$, middle dose, $20 \mu \mathrm{M}$, high dose, $200 \mu \mathrm{M}$; glyceric acid, palmitic acid, stearic acid, alanine, GA, hydroglutaric acid, taurine, and 2-aminoisobutyric acid $=$ low dose, $20 \mu \mathrm{M}$, middle dose, $200 \mu \mathrm{M}$, high dose, $2 \mathrm{mM}$; 3-hydrobutyric acid = low dose, $30 \mu \mathrm{M}$, middle dose, $300 \mu \mathrm{M}$, high dose, $3 \mathrm{mM}$; urea = low dose, $2 \mathrm{mM}$, middle dose, $20 \mathrm{mM}$, high dose, $200 \mathrm{mM}$. In vitro microsomal and recombinant human UGT incubation systems were performed as previously described elsewhere (Maul et al., 2015; Song et al., 2015). MPA (1 mM) was used as the probe substrate of UGT1A7 to evaluate the activity of microsomes and recombinant UGT1A7 enzyme (Mohamed et al., 2008).

Because BRB could also be metabolized well by recombinant human UGT1A9, 1A1, 1A8, or 1A3 (data not shown), BRB (50 $\mu \mathrm{M})$ was selected as the probe substrate of those four isoforms. Valproic acid (1 $\mathrm{mM}$ ) was selected for UGT1A4 and 1A10 (Argikar and Remmel, 2009), and 4-methylumbelliferone (1 mM) was selected for UGT1A6, 2B4, and 2B7 (Udomuksorn et al., 2007; Zhu et al., 2016). The microsomal incubation system was performed in the same way as described earlier. The concentrations of different recombinant human UGTs and the incubation time were selected following the instruction recommendations.

Effects of Linoleic Acid and Glutaric Acid on the Glucuronidation of BRB in Human Renal Proximal Tubular Cells and Mice. Human renal proximal tubular cells (HK-2) were purchased from the China Center for Type Culture Collection (CCTCC, Shanghai, People's Republic of China) and cultured in Dulbecco's modified Eagle's medium/Ham's F-12 medium with $10 \%$ fetal serum. The cells were then cultured in 12-well plates and exposed to $50 \mu \mathrm{M}$ BRB with or without the administration of LA $(200 \mu \mathrm{M})$ and GA $(2 \mathrm{mM})$ for 12 hours at $37^{\circ} \mathrm{C}$.
C57BL/6J mice were randomly divided into three groups: BRB, $\mathrm{BRB}+\mathrm{LA}$, and BRB + GA. Mice in the BRB group were orally administered with a single dose of $B R B(50 \mathrm{mg} / \mathrm{kg})$, while mice in the $\mathrm{BRB}+\mathrm{LA}$ and BRB + GA groups were coadministered with a single dose of LA ( $4 \mathrm{mg} / \mathrm{kg}$, i.p.) or GA (20 mg/kg, i.p.). Urine samples were collected in the metabolic cages for 24 hours.

LC-MS/MS Conditions. The sample analysis work was performed based on an LC-MS/MS system: a Shimadzu Ultra Performance LC-20A system (Shimazu Corpo-ration, Kyoto, Japan) coupled with an API 4000 Triple Quadrupole Mass Spectrometer (AB Sciex, Framingham, MA). A Turbo ion-spray source (for electrospray ionization) was used.

An Agilent (Santa Clara, CA) Zorbax Eclipse Plus C18 column $(2.1 \times 50 \mathrm{~mm}, 3.5 \mu \mathrm{m}$ particle size $)$ was used for chromatographic separation. Mass spectrometry detection was conducted in the positive mode and the source parameters were set as follows: spray voltage, $5500 \mathrm{~V}$; curtain gas, $30 \mathrm{Arb}$; ion source gas 1, $70 \mathrm{Arb}$; ion source gas 2, $70 \mathrm{Arb}$. Compounds were detected in multiple reaction monitoring conditions. The transitions of $m / z 322.4 \rightarrow 307.1$ (declustering potential [DP]: $80 \mathrm{~V}$; collision energy [CE]: $34 \mathrm{eV}), \mathrm{m} / z$ $498.3 \rightarrow 322.1$ (DP: $80 \mathrm{~V}, \mathrm{CE}: 30 \mathrm{eV}$ ), $\mathrm{m} / z$ 321.0 $\rightarrow 207.0$ (DP: $80 \mathrm{~V}, \mathrm{CE}$ : $30 \mathrm{eV}$ ), m/z 514.3 $\rightarrow 321.0$ (DP: $50 \mathrm{~V}, \mathrm{CE}: 25 \mathrm{eV}$ ), $\mathrm{m} / z$ 321.2 $\rightarrow 321.2$ (DP: $80 \mathrm{~V}, \mathrm{CE}: 5 \mathrm{eV}$ ), m/z $353.0 \rightarrow 177.0$ (DP: $50, \mathrm{CE}: 25 \mathrm{eV}$ ), and $339.800 \rightarrow 176.2$ (DP: 80 , CE: $35 \mathrm{eV}$ )were monitored for BRB, BRBG, MPA, MPAG, valproic acid glucuronide, 4-methylumbelliferyl- $\beta$-Dglucuronide, and tetrahydroberberine (IS), respectively.

Statistical Analysis. Data were analyzed with Graph Pad Prism 5.01 (GraphPad Software, La Jolla, CA). Multiple groups of one-factor experiments were assessed using one-way analysis of variance, followed by a Tukey post hoc multiple comparison test. A two-tailed unpaired Student's $t$ test was used in the comparison of two groups. $P<0.05$ was considered a statistically significant difference. Data were expressed as mean \pm S.D., and each group had at least three experiments performed in triplicate.

Partial least squares discriminant analysis (PLS-DA), a method of using partial least squares regression in the discriminant analysis in SIMCA-P+ 13.0 (Umetrics, Umeå, Sweden). Briefly, the data matrix was constructed using compound index as variable names, sample ID as observations, and normalized peak areas as variables. All data were mean-centered and unit variance scaled. The goodness of fit for a model was evaluated using three quantitative parameters based on cross-validation: $R^{2} X$, the explained variation in $X ; R^{2} Y$, the explained variation in $\mathrm{Y} ; \mathrm{Q}^{2} \mathrm{Y}$, the predicated variation in $\mathrm{Y}$. The number of principal components was determined once the $\mathrm{Q} 2 \mathrm{Y}$ value decreased continuously. The range of these parameters is between 0 and 1 ; the closer they approach 1 , the better they could predict or explain.

\section{Results}

HFD Decreased the Ratio of BRBG to BRB in Urinary Excretion. A simultaneous assay of BRB and BRBG was developed using an LC-MS/MS analytical approach based on our previous studies (Yang et al., 2017a). The plasma concentration-time curves and urinary excretion of both BRB and BRBG in the three groups (CSB, CMB, and HMB) were investigated. As shown in Fig. 1, A-D, the HFD slightly increased the mean plasma area under the curve calculated to the last measured concentration $\left(\mathrm{AUC}_{0_{-t} \mathrm{t}}\right)$ of both $\mathrm{BRB}$ and BRBG in the HMB group compared with the CMB group and did not show an obvious influence on the ratio of BRBG to BRB. BRBG in the CSB group showed shoulder peaks, which indicates the possibility of enterohepatic circulation and metabolic interconversion because BRBG could be hydrolyzed via the gut flora. The enterohepatic circulation may decelerate the excretion of BRB and BRBG in the CSB group. As a primary metabolite of berberine, BRB may have a 
A

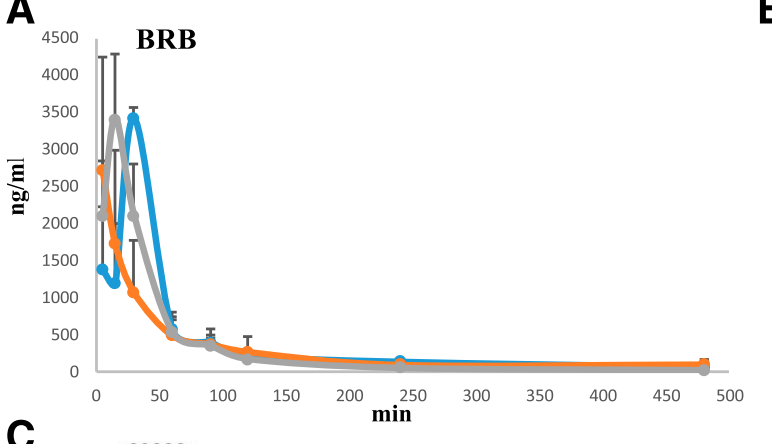

C

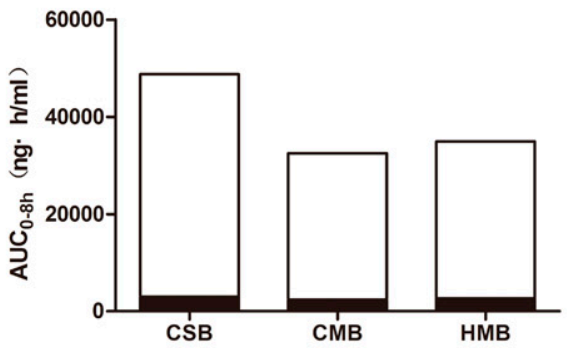

B
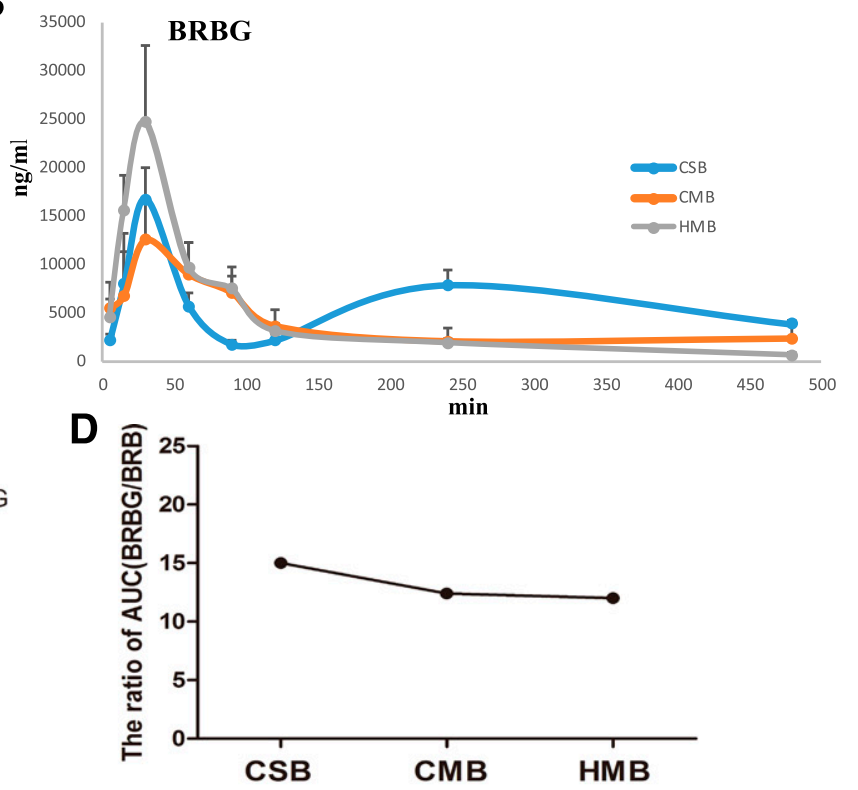

F

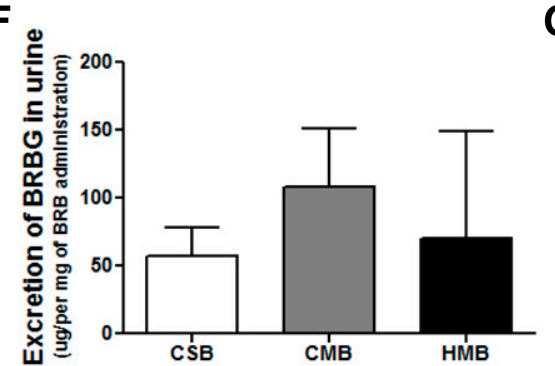

G

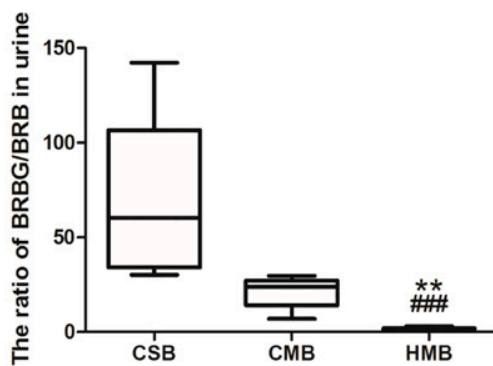

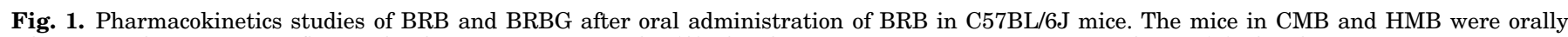

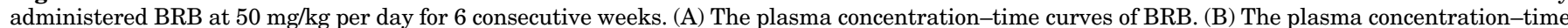

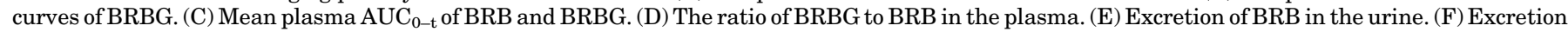

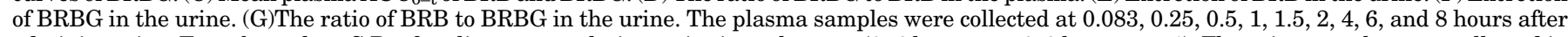

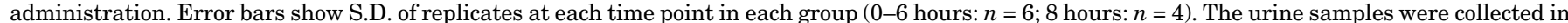

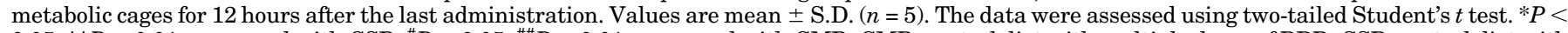

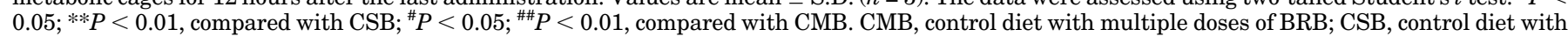
single dose of BRB; HMB, high-fat diet with multiple doses of BRB.

similar antibacterial effect. Therefore, the shoulder peaks were not observed in multiple dose groups.

Interestingly, although the excretion of BRBG in the HMB and CMB groups into urine did not show any significant difference, the ratio of $B R B G$ to $B R B$ significantly decreased in the $\mathrm{HMB}$ group compared with the CMB group because the excretion of BRB was significantly higher in the HMB group (Fig. 1, E-G). The ratio in the CMB group is lower than that in the CSB group. However, there is only marginal statistical significance between these two groups $(P=0.051)$. These results indicate that HFD could significantly increase the urinary excretion of BRB and decrease the ability of glucuronidation. Moreover, pharmacokinetic studies showed BRBG is at an extremely low level in the bile of mice, which suggests a much lower level of glucuronidation metabolism of BRB in the liver (data not shown). In addition to the liver, the glucuronidation process in the kidneys is also at an extremely high level. Some studies have reported that the activity of UGTs in the kidneys is second only to that in the liver (Lohr et al., 1998; Kerdpin et al., 2008; Mutsaers et al., 2013). The inconsistency of glucuronidation proportion of BRB in the plasma and urine indicates that the kidneys may play an important role in BRB glucuronidation.
HFD Slightly Increased Hepatic Glucuronidation but Markedly Decreased Renal Glucuronidation of BRB. In the process of glucuronidation, compounds (containing polar groups, i.e., hydroxyl, amine, carboxyl, etc.) are conjugated with uridine-5' -diphospho- $\alpha$-D-glucuronic acid (UDP-GLcA) under the catalysis of UGT isoforms. UDP-glucose 6-dehydrogenase (UGP), and UDP-glucose 6-dehydrogenase (UGDH) are two essential enzymes that catalyze glucose-1-phosphate to form UDP-GLcA. As our previous study indicated, the overall ability of glucuronidation was decreased because the ratio of BRBG to BRB decreased significantly after HFD combined with BRB administration. We investigated the correlative factor involved in the glucuronidation process to uncover the characteristics of glucuronidation after treatment. No significant difference was observed in the levels of renal and hepatic UDP-GLcA in HB compared with $\mathrm{H}$ or $\mathrm{CB}$ in spite of the inconsistent changes of UGP and UGDH in the liver and kidney after treatment (Fig. 2, A-C).

To further confirm the activities of UGTs after BRB treatment on HFD, hepatic and renal microsomes of different groups were isolated, prepared, and then incubated with BRB in vitro. Based on the rate of $B R B G$ formation in the liver, 


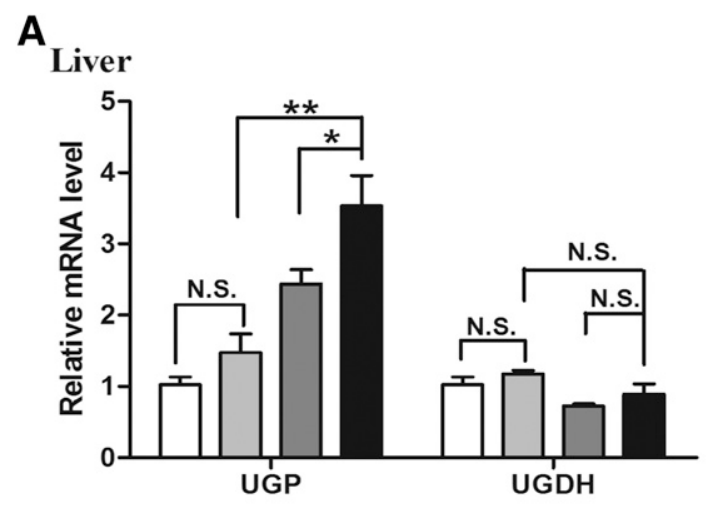

B
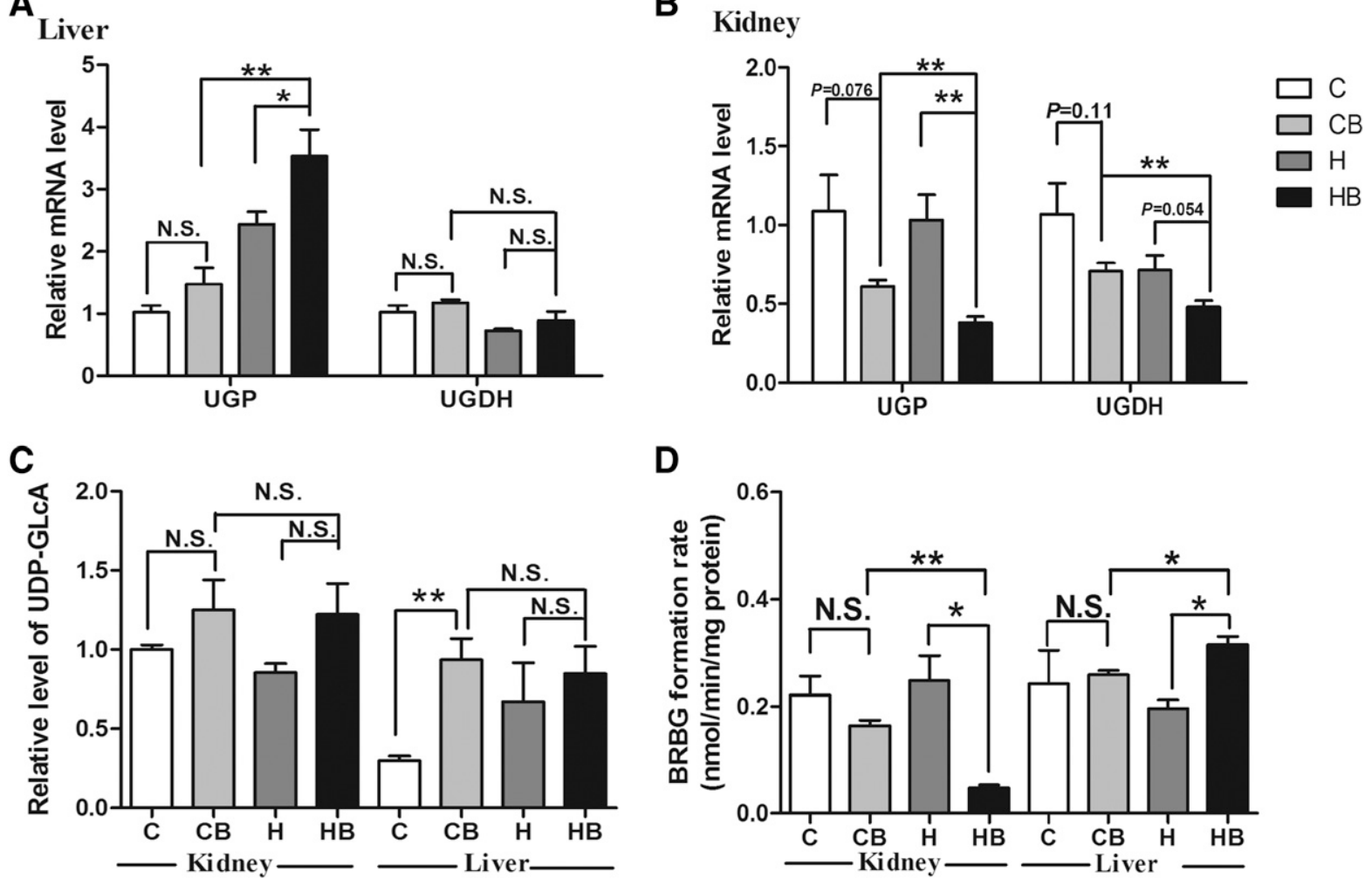

Fig. 2. The alteration of hepatic and renal glucuronidation pathway after treatment. Mice were treated for 6 consecutive weeks. (A) Relative mRNA levels of UGP and UGDH in the liver $(n=5$ ). (B) Relative mRNA levels of UGP and UGDH in the kidneys $(n=5)$. (C) Relative amount of UDP-GLcA in the kidneys and liver $(n=4)$. (D) Activities of UGT isoforms in the renal and hepatic microsomes prepared from tissues of different groups. The rate was determined according to the formation of BRBG after incubation with BRB in vitro $(n=6)$. Values are mean \pm S.D. Two-tailed unpaired Student's $t$ test was used in the comparison of two independent groups. ${ }^{*} P<0.05 ;{ }^{*} P<0.01$. N.S., not statistically significant. C, normal standard diet with vehicle CMC-Na; $\mathrm{CB}$, normal standard diet with BRB $(50 \mathrm{mg} / \mathrm{kg}$ per day, intragastric gavage); $\mathrm{H}$, high-fat diet with vehicle CMC-Na; HB, high-fat diet combined with $\mathrm{BRB}$ (50 mg/kg per day, intragastric gavage).

UGT isoforms in the HB group showed higher activity than in the $\mathrm{CB}$ and $\mathrm{H}$ groups (HB vs. $\mathrm{CB}, P<0.05$; $\mathrm{HB}$ vs. $\mathrm{H}, P<0.05$ ) (Fig. $2 \mathrm{D})$. These results demonstrate the elevated hepatic glucuronidation ability after HFD combined with BRB treatment.

To interpret the paradox between decreased overall glucuronidation ability and increased hepatic glucuronidation ability, the glucuronidation processing in the kidney was also determined. The rate of BRBG formation in renal microsomes is similar to that in hepatic microsomes, which indicated the important role of kidney in the glucuronidation of BRB. Interestingly, in comparison with the liver, the renal glucuronidation ability in the HB group significantly decreased (HB vs. $\mathrm{CB}, P<0.01$; $\mathrm{HB}$ vs. $\mathrm{H}, P<0.05$ ). In conclusion, the amount of renal UDP-GLcA showed no significant difference despite the decreased relative levels of UGP and UGDH, but there was a distinctly decreased renal UGT activity in the renal microsomes of $\mathrm{HB}$, which may contribute to the decreased overall glucuronidation ability.

As an important nuclear receptor, peroxisome proliferatoractivated receptor $\alpha(\operatorname{PPAR} \alpha)$ recently has been reported to regulate the expression and activity of UGT isoenzymes (Xu et al., 2012; Zhou et al., 2013). To further investigate the potential mechanism of renal and hepatic UGT metabolism differences, we determined the relative mRNA levels of Ppar $\alpha$ and its targeting genes, including fatty acid-binding protein (Fabp) and acyl-coenzyme A oxidase (Acox) (Supplemental Fig. 1). There was no significant difference in hepatic Ppar $\alpha$ or the relative amount of its targeting genes after treatment (HB vs. H). In contrast, there was a significant decrease in the renal Ppar $\alpha$ signaling pathway after BRB treatment on HFD, which may be correlative with the decreased activity of UGTs measured before. These results suggested that there was a specific down-regulation of the renal UGT signaling pathway after BRB treatment combined with HFD. Interestingly, in vitro studies indicated that neither BRB nor BRBG exerted a significant inhibitory effect on the Ppar $\alpha$ signaling pathway in renal cells (HK-2), which suggests the existence of some endogenous factors (Supplemental Fig. 2).

UGT Isoenzymes Related to the Glucuronidation of BRB in C57BL/6J Mice. Because HFD combined with BRB greatly inhibited renal UGT activity, we examined the differential effects on hepatic and renal UGTs in C57BL/6J mice and profiled the relative mRNA levels of different UGT isoenzymes in the liver and kidneys, considering the lack of commercial antibodies for mouse UGT isoenzymes. As shown in Fig. 3, A and C, there was an obvious tissue specificity of the main UGT isoenzymes.

In the mouse kidneys, Ugt1a7c showed the absolutely highest mRNA level, which was almost 700-fold higher than that of Ugt2b35, and the mRNA levels of other UGT isoenzymes (1a6, 1a2, 1a1, 1a9, and 2b1) were also at a relatively low level except for Ugt2b5. The UGT distribution in the kidneys is in accordance with previous studies (Buckley and Klaassen, 2007). 


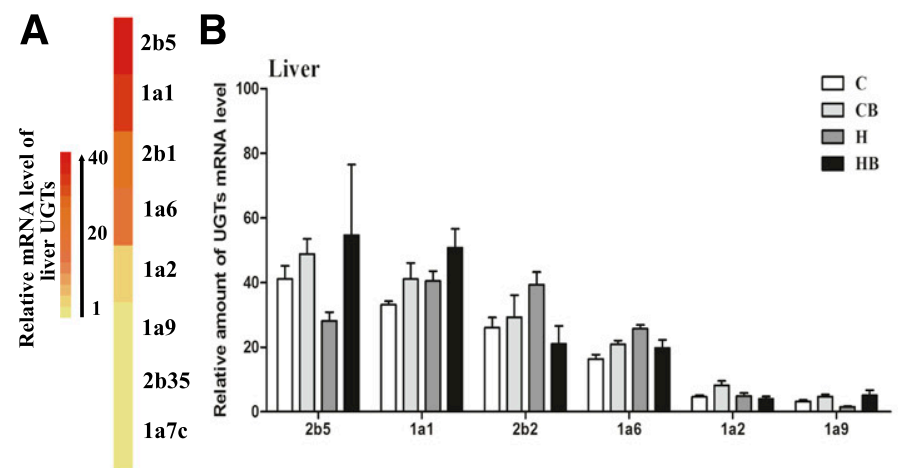

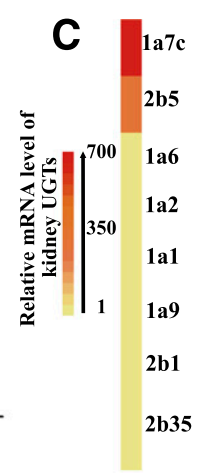

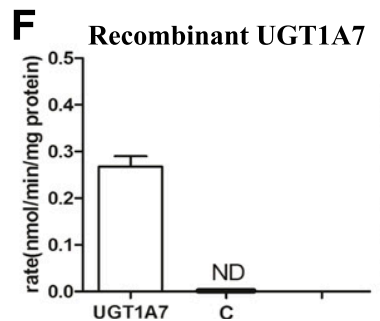

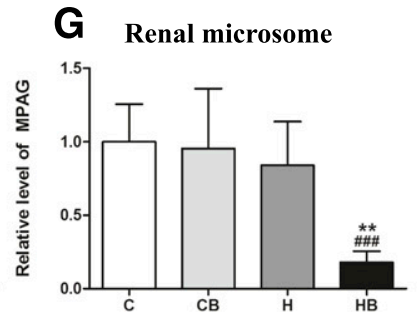

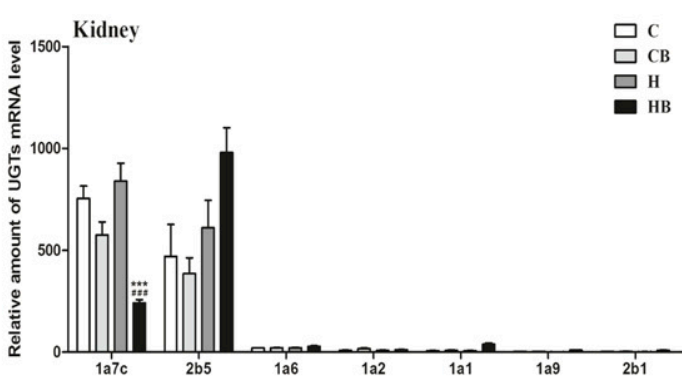

E

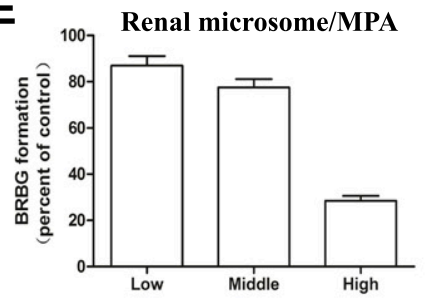

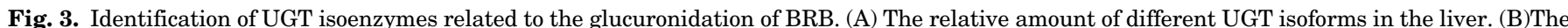

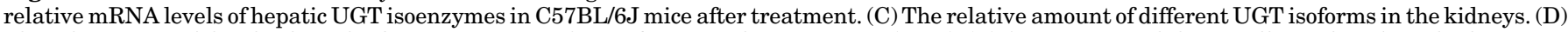

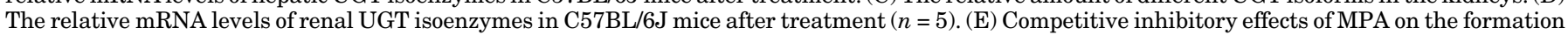

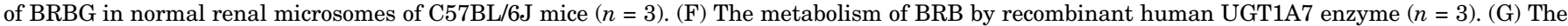

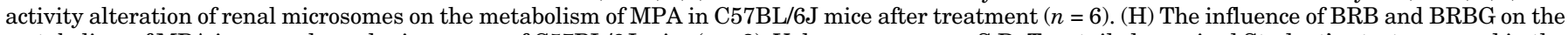

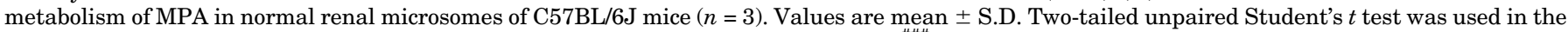
comparison of two independent groups. ${ }^{* *} P<0.01$; ${ }^{* *} P<0.001$, compared with $\mathrm{CB}$; ${ }^{* \#} P<0.001$, compared with $\mathrm{H}$.

In the liver, however, the relative mRNA level of Ugt1a7c was at the lowest level and was nearly 40-fold lower than that of $U g t 2 b 5$, which showed the highest hepatic UGT expression. Moreover, the relative amounts of hepatic Ugt1a1, 2b1, and $1 a 6$ were also relatively high, but $\operatorname{Ugt1a} 2,1 a 9$, and $2 b 35$ were at very low levels. After the HFD combined with BRB treatment, there was a distinct decrease in renal $U g t 1 a 7 c$, which was the highest expressed UGT in the kidneys (Fig. 3D). In the liver, the UGT isoenzymes that expressed at relatively high levels had no significant difference in $\mathrm{HB}$ (vs. $\mathrm{CB}$ or $\mathrm{H}$ ) (Fig. 3B).

Although the lack of commercial antibodies and recombinant enzymes for mouse UGT isoforms limits the investigation on mouse UGT isoenzymes only at mRNA levels, these results were in accordance with the renal and hepatic UGT activities determined before, as shown in Fig. 2D.

There is a high degree of similarity and homology in terms of the genes and metabolic specificity of UGTs between human and rodents (Hanioka et al., 2001; Williams et al., 2002; Mackenzie et al., 2005; Antonilli et al., 2008; Xie et al., 2013). MPA was used as the potential probe substrate of Ugt1a7c to determine the competitive inhibitory effects on the renal glucuronidation of BRB (Inoue et al., 2007; Zhang et al., 2013).

Different concentrations of MPA were incubated with $50 \mu \mathrm{M}$ $\mathrm{BRB}$ in the normal mouse renal microsomal system. The formation of BRBG significantly decreased after incubation with high concentrations of MPA, which indicates that the decreased renal glucuronidation of BRB may be highly related to Ugt1a7c, as shown in Fig. 3E.

Moreover, we also investigated the glucuronidation of BRB under Ugt2b using different concentrations of naloxone as a probe substrate in mouse renal microsomes (Supplemental Fig. 3) (Zhou et al., 2013; Wang et al., 2015). It has been suggested that Ugt2b is not the important enzyme for the glucuronidation of BRB. Hence, the slight increase in Ugt2b5 in $\mathrm{HB}$ should not influence the glucuronidation of BRB, which is consistent with decreased glucuronidation ability.

In addition, BRB could also be metabolized by the recombinant human UGT1A7 enzyme, as shown in Fig. 3F. Because UGT genes share high homology between humans and rodents (Mackenzie et al., 2005), the decreased Ugt1a7c may be highly responsible for the decreased glucuronidation of $\mathrm{BRB}$, since it is the predominantly abundant isoform in mouse kidneys.

Accordingly, we also investigated the glucuronidation of MPA in the renal microsomes of the $\mathrm{C}, \mathrm{CB}, \mathrm{H}$, and $\mathrm{HB}$ groups, as shown in Fig. 3G. The formation of MPAG in the renal microsomes of the $\mathrm{HB}$ group also decreased significantly (HB vs. $\mathrm{CB}, P<0.01$; HB vs. $\mathrm{H}, P<0.001$ ), which confirmed that the UGT isoform for MPA is similar to that for BRB and that Ugt1a7c would be the most relevant isoform. Besides, neither BRB nor its primary metabolite, BRBG, exerted an inhibitory effect on MPAG formation (Fig. 3H), which indicates the existence of other endogenous factors that influence the activity of UGT isoforms.

Metabolomic Approach to Identifying Endogenous Molecules Potentially Inhibiting Glucuronidation of BRB in Mice. Previous studies have suggested that some uremic toxins could inhibit renal metabolic capacity through interference with glucuronidation (Mutsaers et al., 2013). To explore the potential endogenous factors that influence the activity of renal UGT isoforms and to provide a new strategy for clinical detection of drug interactions, we evaluated the urinary metabolic patterns in $\mathrm{C} 57 \mathrm{BL} / 6 \mathrm{~J}$ mice with gas chromatography/mass spectrometry. A PLS-DA model was created with the samples classified into $\mathrm{C}, \mathrm{CB}, \mathrm{H}$, and $\mathrm{HB}$ groups (Fig. 4A). 
The scores plot revealed obvious differences between those four groups. Intragroup samples were prone to cluster closely, while intergroup samples scattered to different extents. Remarkably, the samples from the HB group showed a distinct shift from those of the $\mathrm{H}$ group, which is consistent with the inhibition rate of UGT activity in the HB group compared with the $\mathrm{H}$ group (Fig. 2D).

The volcano plot showed a statistical difference in the metabolic profiles between the $\mathrm{HB}$ and $\mathrm{H}$ groups (Fig. 4B). Based on the discriminant metabolites identified, we found that urine samples from the HB group showed an obviously increased level of purine and pyrimidine metabolites compared with the $\mathrm{H}$ group, including allantoin, urea, aminoisobutyric acid, dihydrouracil, and alanine. Moreover, the levels of three fatty acids (LA, palmitic acid, and stearic acid), one ketone body (3-hydroxybutyric acid), and several other organic acids (glyceric acid, taurine, GA, and hydroxyglutaric acid) were also significantly elevated (Fig. 4, C-E).

Evaluation of the Inhibitory Effect of the Typical Endogenous Molecules on UGT Activity in Renal Microsomes of Mice. In this study, to further explore the possibility of endogenous factors influencing the activity of renal UGT isoforms, 11 elevated compounds were selected based on previous statistical analyses of urinary metabolic profiles, which contained three end products of purine or pyrimidine metabolism (urea, aminoisobutyric acid, and alanine), four fatty acid metabolism-related compounds
(LA, palmitic acid, stearic acid, and 3-hydroxybutyric acid), and four other organic acids (glyceric acid, taurine, GA, and hydroxyglutaric acid).

The inhibitory effects of those selected compounds on UGT activity were evaluated using renal microsomes of C57BL/6J mice. As shown in Fig. 5, urea, GA, LA, 3-hydroxybutyric acid, and palmitic acid could significantly inhibit the formation of MPAG at the high dose or in a dose-dependent way. The inhibitory rates of these compounds at high doses could reach $73.1 \%, 99.0 \%, 44.0 \%, 43.7 \%$, and $61.6 \%$, respectively, compared with the control group. Additionally, no significant effects were observed of these compounds on the Ppar $\alpha$ signaling pathway (data not shown). These data demonstrate the possibility that the elevated endogenous metabolites in urine may contribute to the decreased glucuronidation ability of BRB in C57BL/6J mice.

Evaluation of the Inhibitory Effect of the Typical Endogenous Molecules on Recombinant Human UGT1A7. Based on the previous evidence, the selected compounds could influence the glucuronidation of MPA in renal microsomes of $\mathrm{C} 57 \mathrm{BL} / 6 \mathrm{~J}$ mice, which may be highly attributed to the inhibitory effect on Ugt1a7c. UGT isoforms show high homology between humans and rodents (Mackenzie et al., 2005). For instance, estradiol is used as the typical substrate for human UGT1A1, rat UGT1A1, and mouse Ugt1a1 (Williams et al., 2002; Antonilli et al., 2008; Wang et al., 2015). Although there is a species difference, we first
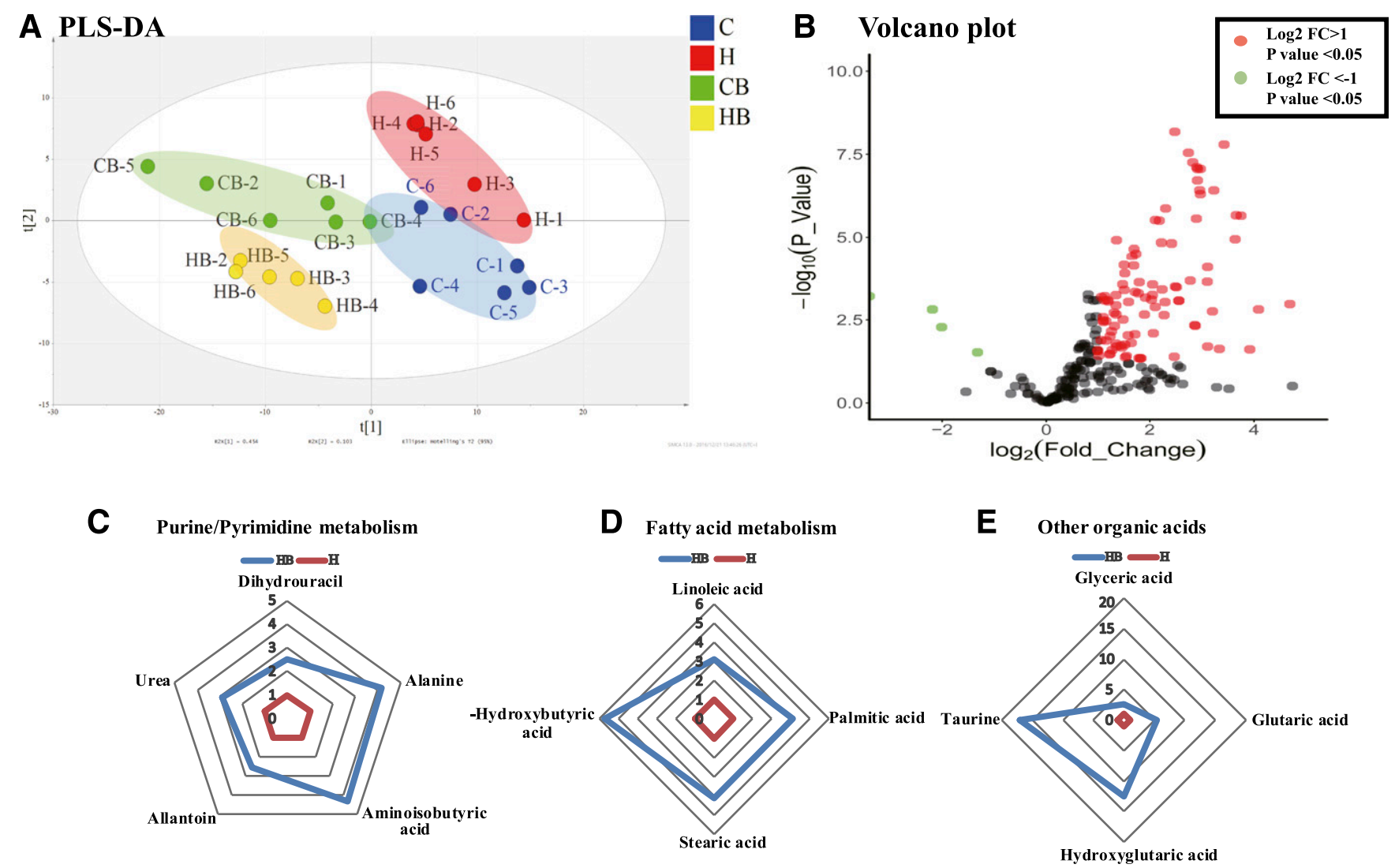

Fig. 4. Metabolomic study of mouse urine after treatment. (A) PLS-DA scores plots. R2X:0.574;R2Y:0.445;Q2Y:0.213. (B) Volcano plot of urinary metabolites in HB compared with $H$. The $P$ values were assessed using two-tailed Student's $t$ test. (C-E) Phase diagrams showed the deviations of the key molecules with significantly elevated levels. The digital labels represent the fold change of the selected molecules in HB group compared with $\mathrm{H}$ group. (C) Metabolites involved in purine/pyrimidine metabolism. (D) Metabolites involved in fatty acid metabolism. (E) Other organic acids. 

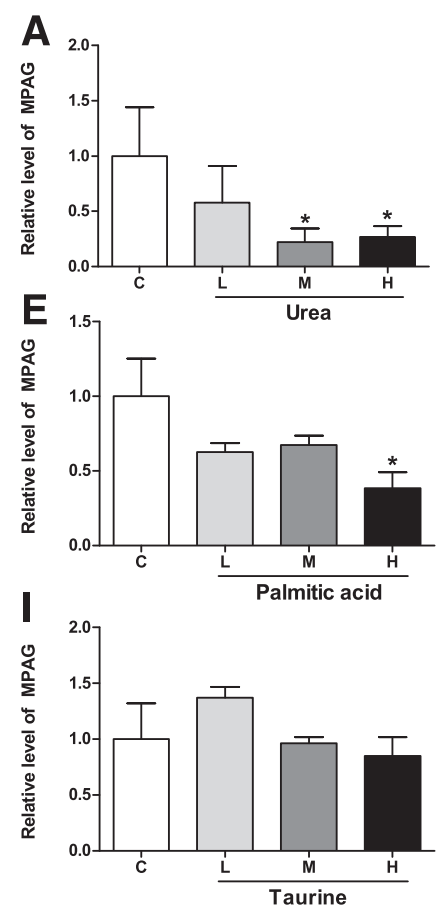
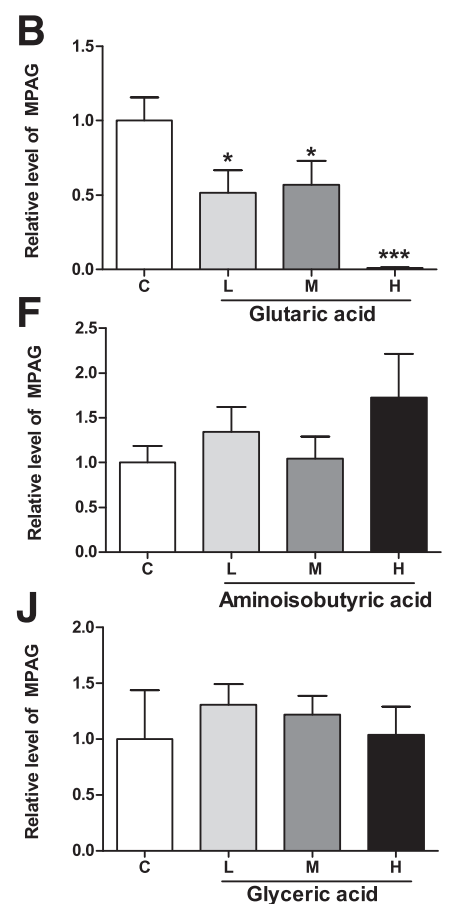
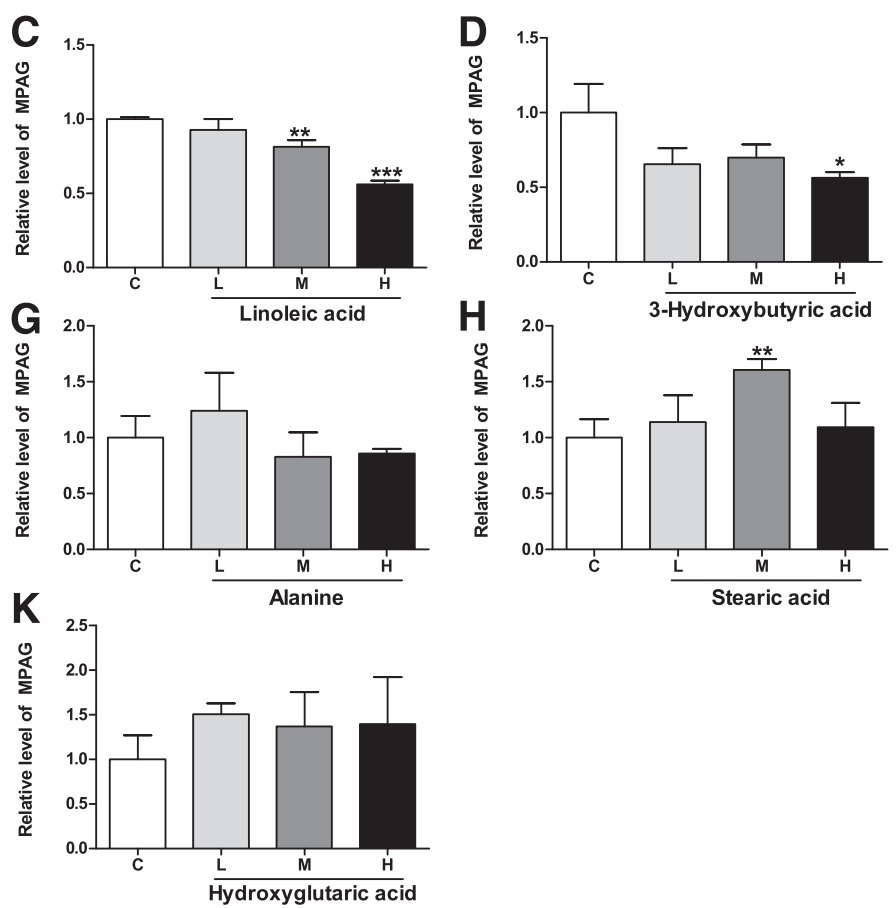

Fig. 5. Evaluation of the inhibitory capability of the typical endogenous molecules on UGT activity in mouse renal microsomes. Eleven elevated compounds were selected and administered in the incubation system of mouse renal microsomes at three different doses for 30 minutes at $37^{\circ} \mathrm{C}$. The relative amount of MPAG formation represents the activity of Ugt1a7c in mouse renal microsomes after treatment. The selected compounds contain (A) urea, (B) glutaric acid, (C) linoleic acid, (D) 3-hydrobutyric acid, (E) palmitic acid, (F) aminoisobutyric acid, (G) alanine, (H) stearic acid, (I) taurine, (J) glyceric acid, and $(\mathrm{K})$ hydroxyglutaric acid. Values are mean \pm S.D. $(n=3)$. The data were assessed by one-way analysis of variance, followed by Tukey post hoc multiple comparison test. $* P<0.05$; $* * P<0.01$; *** $P<0.001$, compared with control. C, blank control group; H, high dose; $\mathrm{L}$, low dose; $\mathrm{M}$, middle dose.

investigated the inhibitory effect of those endogenous compounds on human UGT1A7, as shown in Fig. 6. Remarkably, GA and LA could dose-dependently inhibit the activity of UGT1A7, and the inhibition rates could reach $89.4 \%$ and $48.3 \%$ at high doses, respectively, which are highly consistent with the results from mouse renal microsomes. In contrast, urea, aminoisobutyric acid, alanine, 3-hydroxybutyric acid, palmitic acid, stearic acid, taurine, and glyceric acid did not affect the activity of UGT1A7, and hydroxyglutaric acid only exerted a slight inhibitory effect at low doses. Besides, some of the selected molecules could also influence the expression of UGT1A7 in HK-2 cells (Supplemental Fig. 4). It was suggested that the endogenous molecules are extremely important for influencing both the expression and activity of UGT isoforms.

Linoleic Acid and Glutaric Acid are Potent Candidates for Inhibiting the Glucuronidation of Berberrubine and the Activities of Recombinant Human UGT Isoforms. Because LA and GA could exert direct inhibition on the activity of mouse microsomes and recombinant human UGT1A7 in vitro, we also evaluated their influence on the glucuronidation of BRB in HK-2 cells and mice, as shown in Fig. 7. The inhibition rates of $\mathrm{LA}(200 \mu \mathrm{M})$ and $\mathrm{GA}(2 \mathrm{mM})$ on BRBG formation in HK-2 cells could reach $83.4 \%$ and $55.4 \%$, respectively. The excretion of BRBG in the urine samples decreased significantly after intraperitoneal administration with LA $(4 \mathrm{mg} / \mathrm{kg})$ or GA $(20 \mathrm{mg} / \mathrm{kg})$ in the mice orally administered with a single dose of BRB $(50 \mathrm{mg} / \mathrm{kg})$.

GA and LA were selected as two inhibitory candidates for UGT isoforms. Considering the species difference of UGT isoforms in humans and mice, we systematically evaluated their influence on other human UGT isoforms, which may help to provide some valuable suggestions for clinical studies (Figs. 8 and 9). As the results indicated, GA could also significantly inhibit the activity of UGT1A9 (32.8\%) and 1A8 (12.4\%) at high doses, although the inhibition rates were much lower than that of UGT1A7. Meanwhile, GA could slightly induce UGT1A3 at high doses. No significant influences were observed in the groups for UGT1A1, 1A4, 1A6, 1A10, 2B4, or 2B7. Linoleic acid showed powerful inhibition on UGT1A9 and 1A8. The inhibition rates could even reach $99.3 \%$ for UGT1A9 and $46.8 \%$ for UGT1A8 at $200 \mu \mathrm{M}$. Moreover, LA at the middle dose also significantly inhibited UGT1A9 by $36 \%$. Meanwhile, LA could dose-dependently inhibit the activity of UGT1A1, an important UGT isoform for metabolizing bilirubin and irinotecan.

Importantly, according to the reports from the HMDB, the adopted doses of GA and LA could be achieved in the urine or blood samples of some individuals (HMDB00673, HMDB00661). It is suggested that the levels of endogenous compounds, such as LA and GA, would be closely related to some clinical diseases and drug toxicities resulting from metabolic disturbances.

\section{Discussion}

The glucuronidation process in vivo could be influenced by the alteration of UGT expression (gene or protein) and their enzyme activity. Exogenous or endogenous molecules could directly affect the enzyme activity through bonding with the protein domains. According to our data, the selected endogenous molecules could directly inhibit the UGT activity in an in vitro microsomal or recombinant UGT system. Among these 

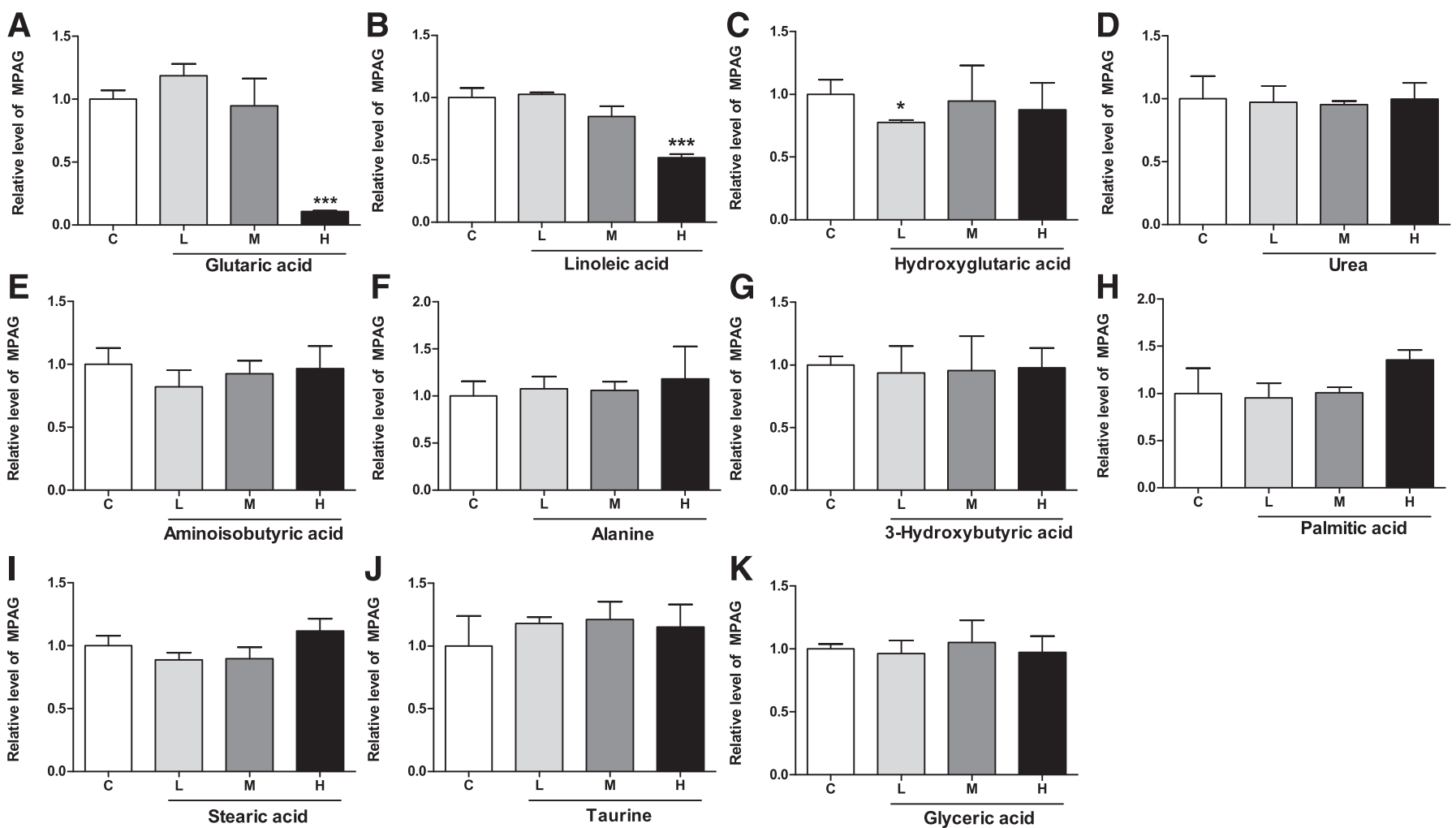

Fig. 6. Evaluation of the inhibitory capability of the typical endogenous molecules on recombinant human UGT1A7. Recombinant human UGT1A7 was incubated with 11 selected compounds at three different doses for 30 minutes $\left(37^{\circ} \mathrm{C}\right)$. The relative amount of MPAG formation represents the activity of UGT1A7. The selected compounds contain (A) glutaric acid, (B) linoleic acid, (C) hydroxyglutaric acid, (D) urea, (E) aminoisobutyric acid, (F) alanine, (G) 3-hydrobutyric acid, (H) palmitic acid, (I) stearic acid, (J) taurine, and (K) glyceric acid. Values are mean \pm S.D. $(n=3)$. The data were assessed by one-way analysis of variance, followed by Tukey post hoc multiple comparison test. ${ }^{*} P<0.05 ; * * * P<0.001$, compared with control. C, blank control group; H, high dose; L, low dose; $\mathrm{M}$, middle dose.

selected molecules, urea, 3-hydroxybutyric acid, and palmitic acid could significantly inhibit the glucuronidation of renal microsomes rather than recombinant human UGT1A7. This may be due to the species difference between the protein domains of UGTs and the contributions of other isoenzymes existed in the microsomes. Besides, some of the selected endogenous molecules (i.e., urea, glyceric acid, taurine, etc.) could also exert significant inhibition on the gene expression of UGT1A7 in HK-2 cells.

Generally, inducers or inhibitors could alter the gene expression through the responsive transcription factors, such as PPAR $\alpha$. In our study, the decreased expression of Ugt1a7c in kidney was accompanied by the down-regulated renal Ppara signaling pathway (Supplemental Fig. 1). However, no significant effects on Ppar $\alpha$ signaling pathway were observed in the HK-2 cells treated with these selected molecules. The regulation of these molecules on the gene expression of UGT isoforms may be through other nuclear receptors such as aryl hydrocarbon receptor, constitutive androstane receptor, and pregnane $\mathrm{X}$ receptor (Buckley and Klaassen, 2009), and the down-regulated renal Ppara signaling pathway in vivo could be caused by some other unknown factors.
A

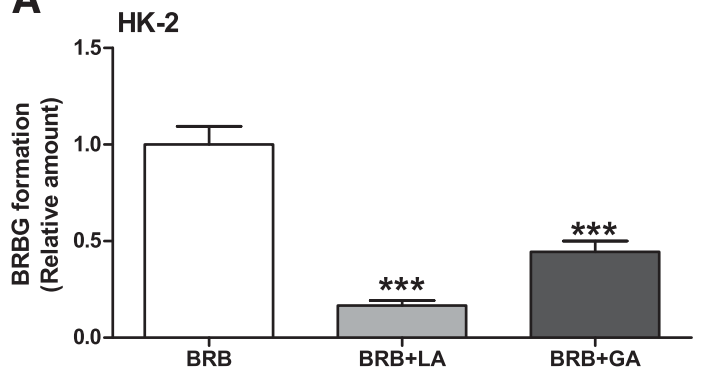

B

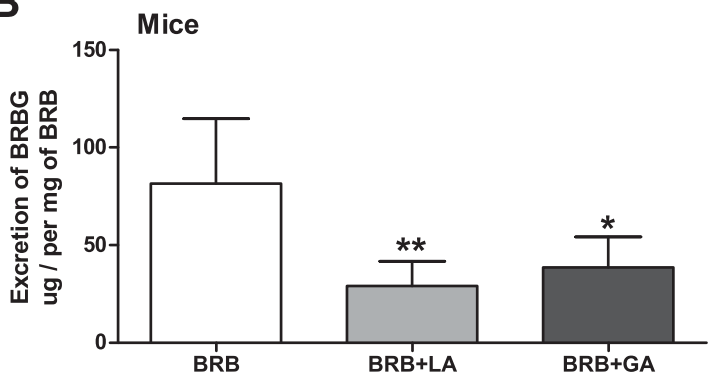

Fig. 7. Effects of linoleic acid (LA) and glutaric acid (GA) on the glucuronidation of BRB in HK-2 cells and mice. (A) Effects of LA and GA on BRBG formation in HK-2 cells. Cells were exposed to $50 \mu \mathrm{M} \mathrm{BRB}$ with or without the administration of LA $(200 \mu \mathrm{M})$ and $\mathrm{GA}(2 \mathrm{mM})$ for 12 hours at $37^{\circ} \mathrm{C}(n=4)$. (B) Effects of LA and GA on BRBG excretion in urine. Mice in the BRB group were orally administered with a single dose of BRB (50 mg/kg), and mice in the BRB + LA and BRB + GA groups were coadministered with a single dose of LA (4 mg/kg, i.p.) or GA ( $20 \mathrm{mg} / \mathrm{kg}$, i.p.). Urine samples were collected in the metabolic cages for 24 hours $(n=5)$. The data were assessed by one-way analysis of variance, followed by Tukey post hoc multiple comparison test. $* P<$ $0.05 ; * * P<0.01 ; * * P<0.001$, compared with BRB group. 
A

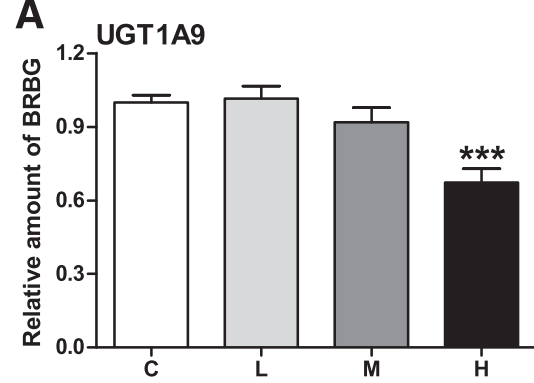

D

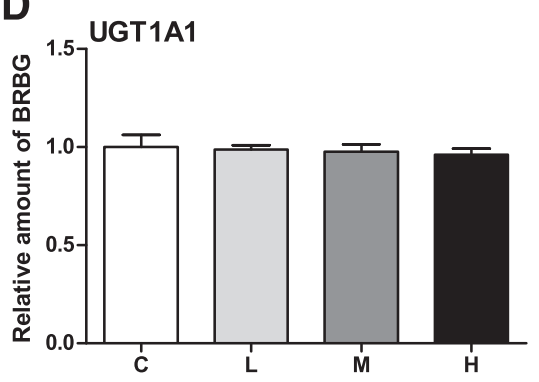

G

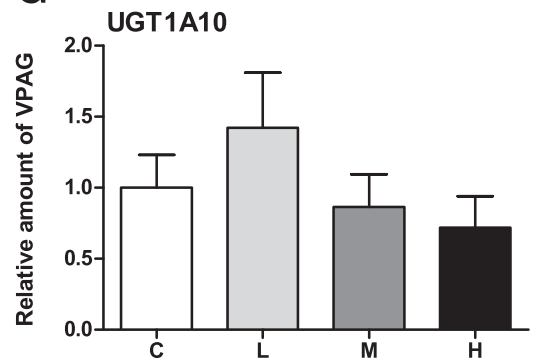

B

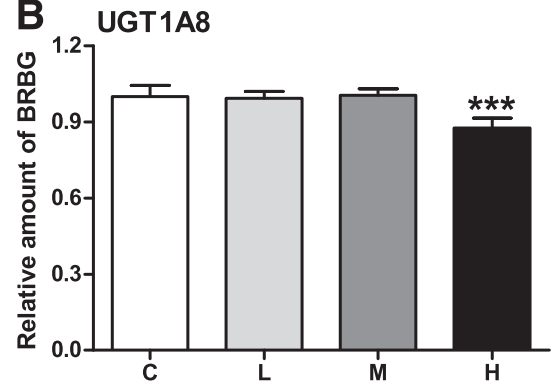

E

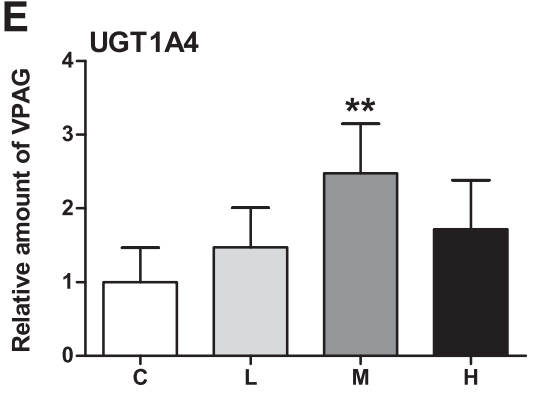

$\mathrm{H}$

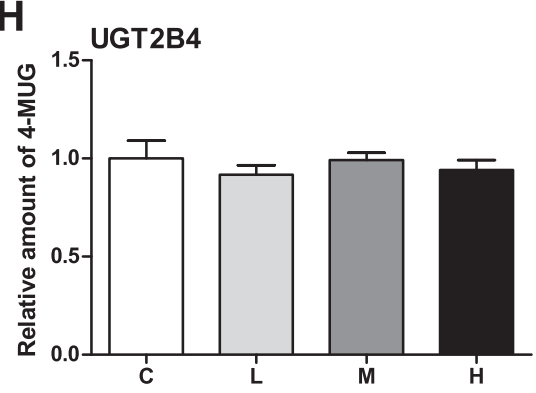

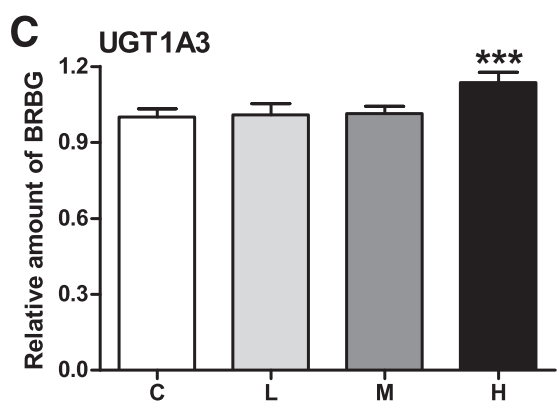

$\mathbf{F}$

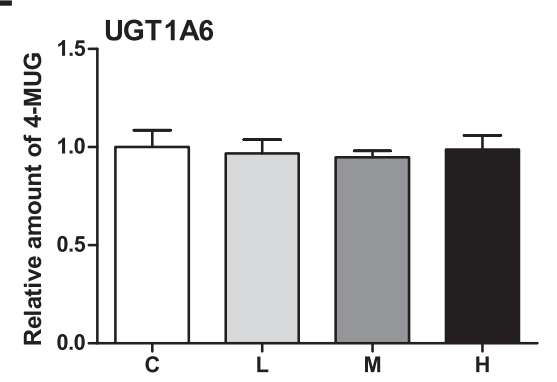

I

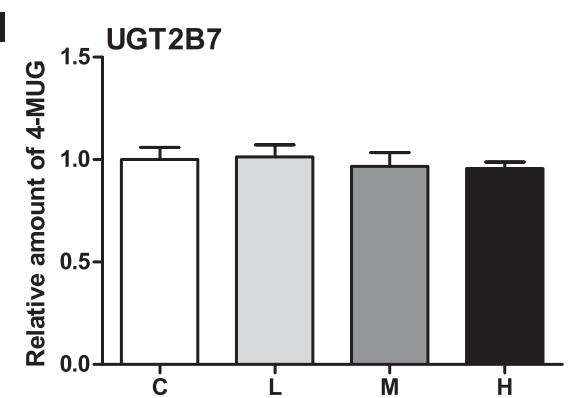

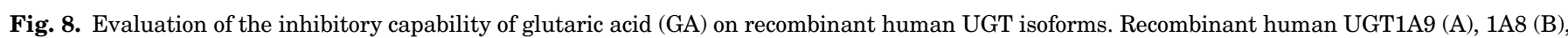

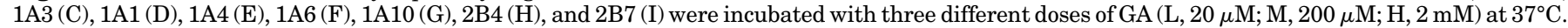

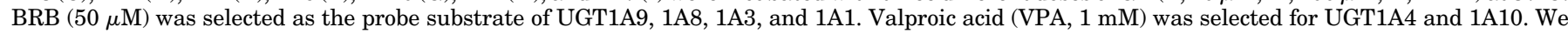

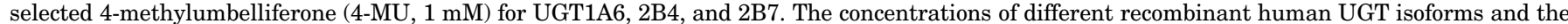

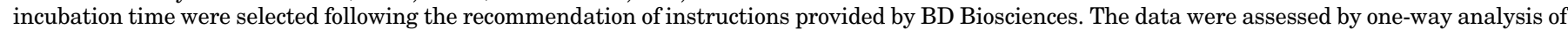
variance, followed by Tukey post hoc multiple comparison test. $* * P<0.01$; $* * * P<0.001$, compared with control.

Ugt1a7c is a predominantly abundant isoform in mouse kidneys, and UGT1A7 is also highly expressed in human kidneys (Harbourt et al., 2012). In our study, recombinant human UGT1A7 was employed and compared with mouse Ugt1a7c because they possess high similarity and homology. However, due to the existence of species difference, Ugt1a7c and UGT1A7 may possess different metabolic properties. Moreover, UGT1A7 is involved in the metabolism of some clinical drugs and xenobiotics, such as acetaminophen and benzo(a)pyrene (Maruo et al., 2005). In Fig. 6, GA and LA were found to exert a significant inhibitory effect on UGT1A7. Thus, the altered levels of them in vivo might possibly contribute to the perturbation on glucuronidation through UGT1A7, which may result in the alteration of glucuronidation capacity in the kidneys. Previous evidence has indicated that the UGT family shares a high degree of similarity in terms of gene and protein structure (de Wildt et al., 1999; Tukey and Strassburg, 2000; Yang et al., 2017b). In this study, GA and LA are also proven to significantly inhibit the activity of UGT1A8 and 1A9, as shown in Figs. 8 and 9. UGT1A9 is also an important UGT isoform that is highly expressed in both the liver and kidneys (Ohno and Nakajin, 2009), and it is responsible for the metabolism of numerous clinical drugs.
To date, the influence of endogenous molecules on drug metabolism has been paid less attention as compared with DDIs. There is very little evidence to confirm the effect of endogenous regulators, and those studies were performed merely by employing in vitro microsomal or recombinant enzymes (Tsoutsikos et al., 2004; Fang et al., 2013). No confirmed in vivo results have been obtained of the effects of endogenous molecules on glucuronidation. In our study, for the first time, we demonstrated that an elevation of endogenous molecules-including GA, LA, 3-hydroxybutyric acid, and palmitic acid-could significantly contribute to the inhibition of mouse renal glucuronidation. Among those compounds, GA and LA are the two strongest potential inhibitors for human recombinant UGT1A7, 1A8, and 1A9. Because there is no intact signal pathway available in the in vitro incubation system, the inhibition of the endogenous molecules on the activity of mouse microsomes and human UGT isoforms suggests the direct effect of the molecules on UGT isoforms. Also, for the first time we confirm the inhibitory effects of endogenous LA and GA on the glucuronidation metabolism in human renal proximal tubular cells (HK-2) and in mice, in addition to microsomes and recombinant UGT isoforms. 
A

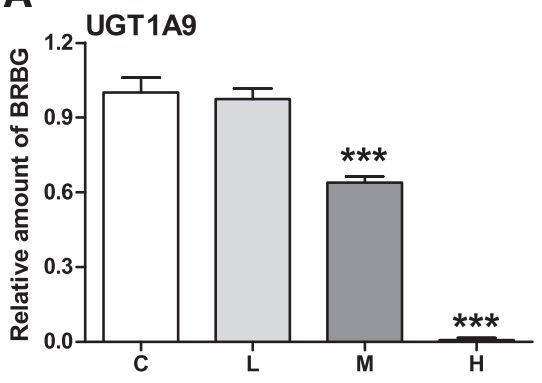

D

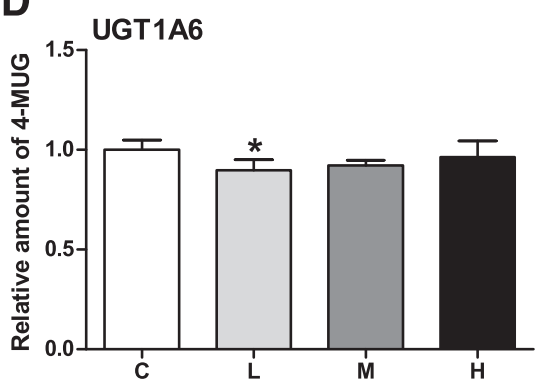

G

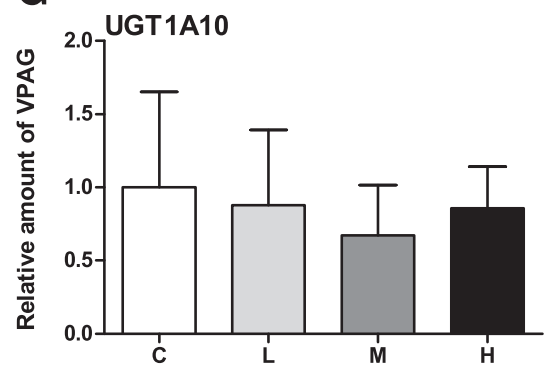

B

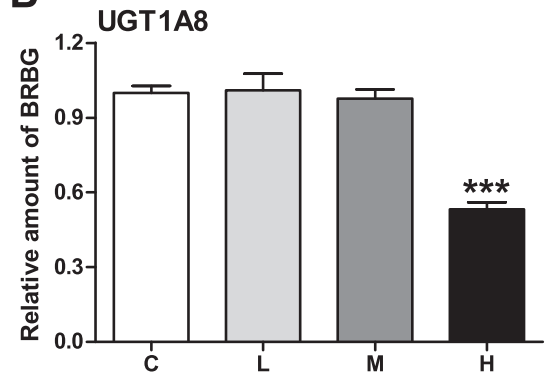

E

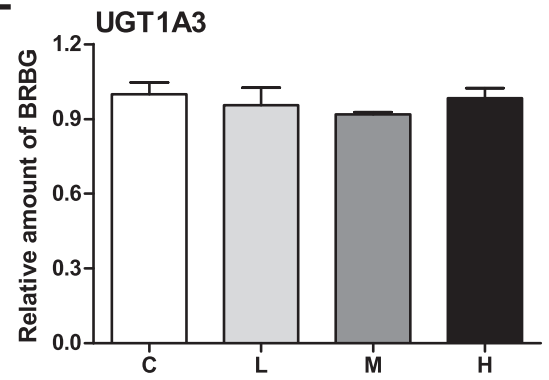

H

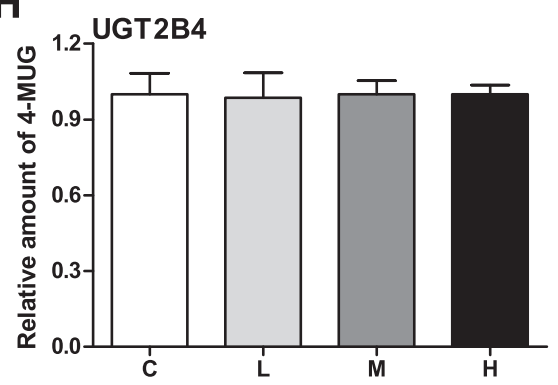

C

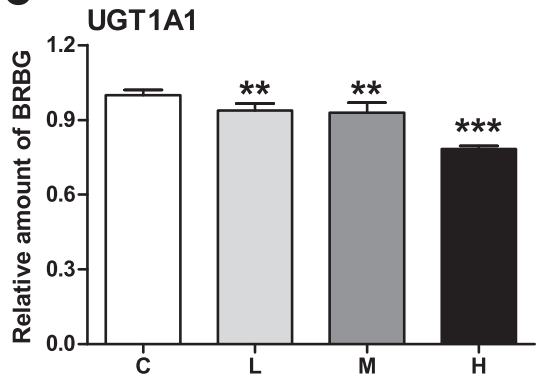

F

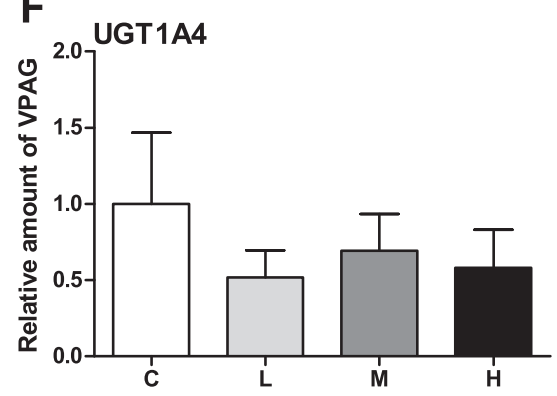

I

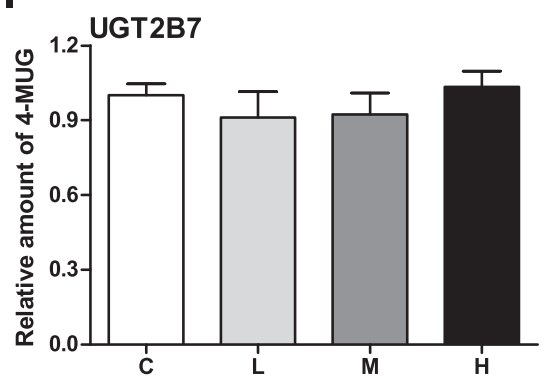

Fig. 9. Evaluation of the inhibitory capability of linoleic acid (LA) on recombinant human UGT isoforms. Recombinant human UGT1A9 (A), 1A8 (B), 1A1 (C), 1A6 (D), 1A3 (E), 1A4 (F), 1A10 (G), 2B4(H), and 2B7 (I) were incubated with three different doses of LA (L, $2 \mu \mathrm{M} ; \mathrm{M}, 20 \mu \mathrm{M} ; \mathrm{H}, 200 \mu \mathrm{M})$ at $37^{\circ} \mathrm{C}$. BRB $(50 \mu \mathrm{M})$ was selected as the probe substrate of UGT1A9, 1A8, 1A3, and 1A1. Valproic acid (VPA, $1 \mathrm{mM}$ ) was selected for UGT1A4 and 1A10. We selected 4-methylumbelliferone (4-MU, $1 \mathrm{mM}$ ) for UGT1A6, 2B4, and 2B7. The concentrations of different recombinant human UGTs and the incubation time were selected following the recommendation of instructions provided by BD Biosciences. The data were assessed by one-way analysis of variance, followed by Tukey post hoc multiple comparison test. $* P<0.05 ; * * P<0.01 ; * * * P<0.001$, compared with control.

The basis of this study was the specific inhibitory effect on renal glucuronidation of $\mathrm{BRB}$ under a HFD. In fact, there may be more endogenous compounds that change during this process. Although the selected molecules proved to exert an inhibitory effect on UGT isoforms, some other endogenous molecules or potential mechanisms may also be involved in the inhibited glucuronidation of BRB seen in this study. Most importantly, this study provided a valuable strategy for employing a metabolomics methodology to identify endogenous molecules on drug-metabolizing enzymes.

Furthermore, the effective concentrations of GA and LA administered could be achieved in normal or abnormal human urine or blood samples according to the reports from HMDB; that is, the actual concentrations of LA in the blood samples of some normal and abnormal individuals are higher than $200 \mu \mathrm{M}$ (HMDB00673), and the urinary levels of GA in some individuals with glutaric aciduria are high than $2 \mathrm{mM}$ (HMDB00661). The data suggested that LA and GA possibly contribute to the individual variation of glucuronidation, and some endogenous molecules might be key factors contributing to individual differences in drug metabolism. Furthermore, a previous study confirmed the inhibitory potential of endogenous bile acids toward cytochrome P450 activity (Chen and Farrell, 1996) and indicated that the increased levels bile acids during the hepatic diseases may interfere with the glucuronidation of xenobiotics such as drugs (Fang et al., 2013). Therefore, the metabolic perturbation in some metabolic diseases, which involve an altered level of these endogenous molecules, may induce variation of glucuronidation of xenobiotics. Importantly, individual variances can be evaluated, and personalized medication can be suggested for the substrates of UGT based on the in vivo levels of the endogenous molecules.

\section{Authorship Contributions}

Participated in research design: Yang, Xie, Wang, Aa.

Conducted experiments: Yang, Li, Yan, Peng.

Performed data analysis: Yang, Sun, He.

Wrote or contributed to the writing of the manuscript: Yang, Wang, Aa.

\section{References}

Aa J, Trygg J, Gullberg J, Johansson AI, Jonsson P, Antti H, Marklund SL, and Moritz T (2005) Extraction and GC/MS analysis of the human blood plasma metabolome. Anal Chem 77:8086-8094.

Antonilli L, Brusadin V, Milella MS, Sobrero F, Badiani A, and Nencini P (2008) In vivo chronic exposure to heroin or naltrexone selectively inhibits liver microsome formation of estradiol-3-glucuronide in the rat. Biochem Pharmacol 76:672-679. 
Argikar UA and Remmel RP (2009) Effect of aging on glucuronidation of valproic acid in human liver microsomes and the role of UDP-glucuronosyltransferase UGT1A4 UGT1A8, and UGT1A10. Drug Metab Dispos 37:229-236.

Atherton JC (2012) Renal blood flow, glomerular filtration and plasma clearance. Anaesth Intensive Care Med 13:315-319.

Bánhegyi G, Braun L, Marcolongo P, Csala M, Fulceri R, Mandl J, and Benedetti A (1996) Evidence for an UDP-glucuronic acid/phenol glucuronide antiport in rat liver microsomal vesicles. Biochem $J$ 315:171-176.

Buckley DB and Klaassen CD (2007) Tissue- and gender-specific mRNA expression of UDP-glucuronosyltransferases (UGTs) in mice. Drug Metab Dispos 35:121-127.

Buckley DB and Klaassen CD (2009) Induction of mouse UDP-glucuronosyltransferase mRNA expression in liver and intestine by activators of aryl-hydrocarbon receptor, constitutive androstane receptor, pregnane $\mathrm{X}$ receptor, peroxisome proliferatoractivated receptor alpha, and nuclear factor erythroid 2-related factor 2. Drug Metab Dispos 37:847-856.

Chen J and Farrell GC (1996) Bile acids produce a generalized reduction of the catalytic activity of cytochromes $\mathrm{P} 450$ and other hepatic microsomal enzymes in vitro: relevance to drug metabolism in experimental cholestasis. J Gastroenterol Hepatol 11:870-877.

Court MH, Zhang X, Ding X, Yee KK, Hesse LM, and Finel M (2012) Quantitative distribution of mRNAs encoding the 19 human UDP-glucuronosyltransferase enzymes in 26 adult and 3 fetal tissues. Xenobiotica 42:266-277.

de Wildt SN, Kearns GL, Leeder JS, and van den Anker JN (1999) Glucuronidation in humans. Pharmacogenetic and developmental aspects. Clin Pharmacokinet 36:439-452.

Fang ZZ, He RR, Cao YF, Tanaka N, Jiang C, Krausz KW, Qi Y, Dong PP, Ai CZ, Sun $\mathrm{XY}$, et al (2013) A model of in vitro UDP-glucuronosyltransferase inhibition by bile acids predicts possible metabolic disorders. J Lipid Res 54:3334-3344

Feere DA, Velenosi TJ, and Urquhart BL (2015) Effect of erythropoietin on hepatic cytochrome P450 expression and function in an adenine-fed rat model of chronic kidney disease. $\mathrm{Br}$ J Pharmacol 172:201-213.

Gradinaru D, Minn AL, Artur Y, Minn A, and Heydel JM (2012) Effect of oxidative stress on UDP-glucuronosyltransferases in rat astrocytes. Toxicol Lett 213:316-324. Gruber M, Bellemare J, Hoermann G, Gleiss A, Porpaczy E, Bilban M, Le T, Zehetmayer S, Mannhalter C, Gaiger A, et al. (2013) Overexpression of uridine diphospho glucuronosyltransferase 2B17 in high-risk chronic lymphocytic leukemia. Blood 121:1175-1183.

Gu S, Cao B, Sun R, Tang Y, Paletta JL, Wu X, Liu L, Zha W, Zhao C, Li Y, et al. (2015) A metabolomic and pharmacokinetic study on the mechanism underlying the lipid-lowering effect of orally administered berberine [published correction in Mol Biosyst (2015) 11:664]. Mol Biosyst 11:463-474.

Guengerich FP (2006) Cytochrome P450s and other enzymes in drug metabolism and toxicity. AAPS J 8:E101-E111.

Guo J, Yong Y, Aa J, Cao B, Sun R, Yu X, Huang J, Yang N, Yan L, Li X, et al. (2016) Compound danshen dripping pills modulate the perturbed energy metabolism in a rat model of acute myocardial ischemia. Sci Rep 6:37919.

Hanioka N, Jinno H, Tanaka-Kagawa T, Nishimura T, and Ando M (2001) Determination of UDP-glucuronosyltransferase UGT1A6 activity in human and rat liver microsomes by HPLC with UV detection. J Pharm Biomed Anal 25:65-75.

Harbourt DE, Fallon JK, Ito S, Baba T, Ritter JK, Glish GL, and Smith PC (2012) Quantification of human uridine-diphosphate glucuronosyl transferase 1A isoforms in liver, intestine, and kidney using nanobore liquid chromatographytandem mass spectrometry. Anal Chem 84:98-105

Inoue K, Miura M, Satoh S, Kagaya H, Saito M, Habuchi T, and Suzuki T (2007) Influence of UGT1A7 and UGT1A9 intronic I399 genetic polymorphisms on mycophenolic acid pharmacokinetics in Japanese renal transplant recipients. Ther Drug Monit 29:299-304.

Kang HE, Sohn SI, Baek SR, Lee JW, and Lee MG (2010) Liquiritigenin pharmacokinetics in a rat model of diabetes mellitus induced by streptozotocin: greater formation of glucuronides in the liver, especially M2, due to increased hepatic uridine 5'-diphosphoglucuronic acid level. Metabolism 59:1472-1480.

Kerdpin O, Knights KM, Elliot DJ, and Miners JO (2008) In vitro characterisation of human renal and hepatic frusemide glucuronidation and identification of the UDP glucuronosyltransferase enzymes involved in this pathway. Biochem Pharmacol 76:249-257.

Kiang TK, Ensom MH, and Chang TK (2005) UDP-glucuronosyltransferases and clinical drug-drug interactions. Pharmacol Ther 106:97-132.

Kua LF, Ross S, Lee SC, Mimura K, Kono K, Goh BC, and Yong WP (2012) UGT1A6 polymorphisms modulated lung cancer risk in a Chinese population. PLoS One 7:e42873.

Li YH, Li Y, Yang P, Kong WJ, You XF, Ren G, Deng HB, Wang YM, Wang YX, Jiang JD, et al. (2010) Design, synthesis, and cholesterol-lowering efficacy for prodrugs of berberrubine. Bioorg Med Chem 18:6422-6428.

Liu M, Wang Q, Liu F, Cheng X, Wu X, Wang H, Wu M, Ma Y, Wang G, and Hao H (2013) UDP-glucuronosyltransferase 1A compromises intracellular accumulation and anti-cancer effect of tanshinone IIA in human colon cancer cells. PLoS One 8:e79172.

Livak KJ and Schmittgen TD (2001) Analysis of relative gene expression data using real-time quantitative PCR and the $2^{-\Delta \Delta \mathrm{C}(\mathrm{T})}$ method. Methods 25:402-408.

Lohr JW, Willsky GR, and Acara MA (1998) Renal drug metabolism. Pharmacol Rev 50: 107-141.

Mackenzie PI, Bock KW, Burchell B, Guillemette C, Ikushiro S, Iyanagi T, Miners JO, Owens IS, and Nebert DW (2005) Nomenclature update for the mammalian UDP glycosyltransferase (UGT) gene superfamily. Pharmacogenet Genomics 15:677-685.

Mannhalter C, Koizar D, and Mitterbauer G (2000) Evaluation of RNA isolation methods and reference genes for RT-PCR analyses of rare target RNA. Clin Chem Lab Med 38:171-177.

Margaillan G, Rouleau M, Fallon JK, Caron P, Villeneuve L, Turcotte V, Smith PC Joy MS, and Guillemette C (2015) Quantitative profiling of human renal UDPglucuronosyltransferases and glucuronidation activity: a comparison of normal and tumoral kidney tissues. Drug Metab Dispos 43:611-619.

Maruo Y, Iwai M, Mori A, Sato H, and Takeuchi Y (2005) Polymorphism of UDPglucuronosyltransferase and drug metabolism. Curr Drug Metab 6:91-99.

Maul R, Warth B, Schebb NH, Krska R, Koch M, and Sulyok M (2015) In vitro glucuronidation kinetics of deoxynivalenol by human and animal microsomes and recombinant human UGT enzymes. Arch Toxicol 89:949-960.
Mohamed MF, Harvey SS, and Frye RF (2008) Determination of mycophenolic acid glucuronide in microsomal incubations using high performance liquid chromatography-tandem mass spectrometry. $J$ Chromatogr B Analyt Technol Biomed Life Sci 870:251-254.

Mutsaers HA, Wilmer MJ, Reijnders D, Jansen J, van den Broek PH, Forkink M, Schepers E, Glorieux G, Vanholder R, van den Heuvel LP, et al. (2013) Uremic toxins inhibit renal metabolic capacity through interference with glucuronidation and mitochondrial respiration. Biochim Biophys Acta 1832:142-150.

Oda S, Fukami T, Yokoi T, and Nakajima M (2015) A comprehensive review of UDP-glucuronosyltransferase and esterases for drug development. Drug Metab Pharmacokinet 30:30-51.

Ohno S and Nakajin S (2009) Determination of mRNA expression of human UDP glucuronosyltransferases and application for localization in various human tissues by realtime reverse transcriptase-polymerase chain reaction. Drug Metab Dispos 37:32-40.

Rowland A, Miners JO, and Mackenzie PI (2013) The UDP-glucuronosyltransferases: their role in drug metabolism and detoxification. Int J Biochem Cell Biol 45:1121-1132.

Song JH, Cui L, An LB, Li WT, Fang ZZ, Zhang YY, Dong PP, Wu X, Wang LX, Gonzalez FJ, et al. (2015) Inhibition of UDP-Glucuronosyltransferases (UGTs) activity by constituents of Schisandra chinensis. Phytother Res 29:1658-1664.

Tephly TR and Burchell B (1990) UDP-glucuronosyltransferases: a family of detoxifying enzymes. Trends Pharmacol Sci 11:276-279.

Tsoutsikos P, Miners JO, Stapleton A, Thomas A, Sallustio BC, and Knights KM (2004) Evidence that unsaturated fatty acids are potent inhibitors of renal UDPglucuronosyltransferases (UGT): kinetic studies using human kidney cortical microsomes and recombinant UGT1A9 and UGT2B7. Biochem Pharmacol 67:191-199.

Tukey RH and Strassburg CP (2000) Human UDP-glucuronosyltransferases: metabolism, expression, and disease. Annu Rev Pharmacol Toxicol 40:581-616.

Udomuksorn W, Elliot DJ, Lewis BC, Mackenzie PI, Yoovathaworn K, and Miners JO (2007) Influence of mutations associated with Gilbert and Crigler-Najjar type II syndromes on the glucuronidation kinetics of bilirubin and other UDP glucuronosyltransferase 1A substrates. Pharmacogenet Genomics 17:1017-1029. van der Bol JM, Loos WJ, de Jong FA, van Meerten E, Konings IR, Lam MH, de Bruijn P, Wiemer EA, Verweij J, and Mathijssen RH (2011) Effect of omeprazole on the pharmacokinetics and toxicities of irinotecan in cancer patients: a prospective cross-over drug-drug interaction study. Eur J Cancer 47:831-838.

Wang H, Yan T, Xie Y, Zhao M, Che Y, Zhang J, Liu H, Cao L, Cheng X, Xie Y, et al. (2015) Mechanism-based inhibitory and peroxisome proliferator-activated receptor $\alpha$-dependent modulating effects of silybin on principal hepatic drug-metabolizing enzymes. Drug Metab Dispos 43:444-454.

Williams JA, Ring BJ, Cantrell VE, Campanale K, Jones DR, Hall SD, and Wrighton SA (2002) Differential modulation of UDP-glucuronosyltransferase 1A1 (UGT1A1) catalyzed estradiol-3-glucuronidation by the addition of UGT1A1 substrates and other compounds to human liver microsomes. Drug Metab Dispos 30:1266-1273.

Xie H, Sun S, Cheng X, Yan T, Zheng X, Li F, Qi Q, Wang G, and Hao H (2013) Dysregulations of intestinal and colonic UDP-glucuronosyltransferases in rats with type 2 diabetes. Drug Metab Pharmacokinet 28:427-434.

Xu J, Kulkarni SR, Li L, and Slitt AL (2012) UDP-glucuronosyltransferase expression in mouse liver is increased in obesity- and fasting-induced steatosis. Drug Metab Dispos 40:259-266.

Yamamura N, Imura-Miyoshi K, and Naganuma H (2000) Panipenum, a carbapenem antibiotic, increases the level of hepatic UDP-glucuronic acid in rats. Drug Metab Dispos 28:1484-1486.

Yang N, Sun RB, Chen XL, Zhen L, Ge C, Zhao YQ, He J, Geng JL, Guo JH, Yu XY, et al. (2017a) In vitro assessment of the glucose-lowering effects of berberrubine-9-O- $\beta$-Dglucuronide, an active metabolite of berberrubine. Acta Pharmacol Sin 38:351-361.

Yang N, Sun RB, Liao X, Aa J, and Wang G (2017b) UDP-glucuronosyltransferases (UGTs) and their related metabolic cross-talk with internal homeostasis: a systematic review of UGT isoforms for precision medicine. Pharmacol Res 121: 169-183.

Yang N, Sun RB, Zhao Y, He J, Zhen L, Guo J, Geng J, Xie Y, Wang J, Feng S, et al. (2016) High fat diet aggravates the nephrotoxicity of berberrubine by influencing on its pharmacokinetic profile. Environ Toxicol Pharmacol 46:319-327.

Yu C, Ritter JK, Krieg RJ, Rege B, Karnes TH, and Sarkar MA (2006) Effect of chronic renal insufficiency on hepatic and renal udp-glucuronyltransferases in rats. Drug Metab Dispos 34:621-627.

Zhang L, Chu X, Wang H, Xie H, Guo C, Cao L, Zhou X, Wang G, and Hao H (2013) Dysregulations of UDP-glucuronosyltransferases in rats with valproic acid and high fat diet induced fatty liver. Eur J Pharmacol 721:277-285.

Zhou X, Xie Y, Qi Q, Cheng X, Liu F, Liao K, Wang G, and Hao H (2013) Disturbance of hepatic and intestinal UDP-glucuronosyltransferase in rats with trinitrobenzene sulfonic acid-induced colitis. Drug Metab Pharmacokinet 28:305-313.

Zhou Y, Cao S, Wang Y, Xu P, Yan J, Bin W, Qiu F, and Kang N (2014) Berberine metabolites could induce low density lipoprotein receptor up-regulation to exert lipid-lowering effects in human hepatoma cells. Fitoterapia 92:230-237.

Zhu L, Xiao L, Li W, Zhang Y, Han W, Zhu Y, Ge G, and Yang L (2016) Human UDPglucuronosyltransferases 1A1, 1A3, 1A9, 2B4 and 2B7 are inhibited by diethylstilbestrol. Basic Clin Pharmacol Toxicol 119:505-511.

Address correspondence to: Dr. Jiye Aa, State Key Laboratory of Natural Medicines, Jiangsu Province Key Laboratory of Drug Metabolism and Pharmacokinetics, Jiangsu Key Laboratory of Drug Design and Optimization, China Pharmaceutical University, Nanjing 210009, People's Republic of China. E-mail: jiyea@cpu.edu.cn or Dr. Guangji Wang, State Key Laboratory of Natural Medicines, Jiangsu Province Key Laboratory of Drug Metabolism and Pharmacokinetics, Jiangsu Key Laboratory of Drug Design and Optimization, China Pharmaceutical University, Nanjing 210009, People's Republic of China. E-mail: guangjiwang@hotmail.com 